\title{
Genome-Based Discovery of Polyketide-Derived Secondary Metabolism Pathways in the Barley Pathogen Ramularia collo-cygni
}

\author{
F. Dussart, ${ }^{1,2,+}$ R. Douglas, ${ }^{1,2}$ E. Sjökvist, ${ }^{1,3}$ P. N. Hoebe, ${ }^{1}$ S. H. Spoel, ${ }^{2}$ and G. R. D. McGrann ${ }^{1, \dagger}$ \\ ${ }^{1}$ Crop and Soil Research Department, SRUC, West Mains Road, Kings Buildings, Edinburgh, EH9 3JG, U.K.; ${ }^{2}$ Institute of \\ Molecular Plant Sciences, School of Biological Sciences, University of Edinburgh, Edinburgh, EH9 3BF, U.K.; and ${ }^{3}$ Institute of \\ Evolutionary Biology, School of Biological Sciences, University of Edinburgh, Edinburgh EH9 3TF, U.K.
}

Accepted 19 March 2018.

\begin{abstract}
Ramularia collo-cygni causes Ramularia leaf spot (RLS) disease of barley. The fungus develops asymptomatically within its host until late in the growing season, when necrotic lesions become visible on upper leaves. Fungal secondary metabolites (SM) have been proposed as important factors in RLS lesion formation but the biosynthetic pathways involved remain largely unknown. Mining the $R$. collo-cygni genome revealed the presence of 10 polyketide synthases (PKS), 10 nonribosomal peptide synthetases (NRPS), and 3 hybrid PKS-NRPS (HPS) identified within clusters of genes with predicted functions associated with secondary metabolism. SM core genes along with their predicted transcriptional regulators exhibited transcriptional coexpression during infection of barley plants. Moreover, their expression peaked during early stages of host colonization and preceded or overlapped with the appearance of disease symptoms, suggesting that SM may manipulate the host to promote colonization or protect $R$. collo-cygni from competing organisms. Accordingly, $R$. collo-cygni inhibited the growth of several fungi in vitro, indicating that it synthesized and excreted antifungal agents. Taken together, these findings demonstrate that the $R$. collo-cygni genome contains the genetic architecture to synthesize a wide range of SM and suggests that coexpression of PKS and HPS is associated with competitive colonization of the host and early symptom development.
\end{abstract}

Ramularia leaf spot (RLS) is a late-season disease of barley (Hordeum vulgare) occurring in temperate regions worldwide. Typical RLS symptoms are often reddish-brown in color, delineated by the leaf vein, surrounded by a chlorotic halo, and visible on both sides of infected leaves (Huss 2002). Symptoms

Current address for G. R. D. McGrann: Potato Branch, Science and Advice for Scottish Agriculture (SASA), Roddinglaw Road, Edinburgh, EH12 9FJ, U.K.

${ }^{\dagger}$ Corresponding authors: F. Dussart; E-mail: Francois.Dussart@sruc.ac.uk; and G. R. D. McGrann; E-mail: grahammcgrann@googlemail.com

Funding: SRUC receives funding from the Scottish Government Rural and Environment Science and Analytical Services Division. S. H. Spoel was funded by Royal Society University Research Fellowships (UF090321 and UF140600).

*The $\boldsymbol{e}$-Xtra logo stands for "electronic extra" and indicates that five supplementary figures and eight supplementary tables are published online. can also be observed on stems, heads, and awns in severely infected crops. As a result, RLS is considered a major disease of barley causing typical yield penalties of approximately $20 \%$ but these losses can be as high as 70\% (Havis et al. 2015). RLS can also reduce grain size by up to $4 \%$, which devalues the crop (Cromey et al. 2002; McGrann and Havis 2017).

RLS is caused by the filamentous fungus Ramularia collocygni, a Dothideomycete species within the order Capnodiales. $R$. collo-cygni was recently confirmed to be closely related to Zymoseptoria tritici, the fungus responsible for Septoria leaf blotch of wheat (Crous et al. 2001; McGrann et al. 2016). $R$. collo-cygni colonization initially occurs asymptomatically before turning to necrotrophic growth (Kaczmarek et al. 2017). The transition from asymptomatic to necrotrophic development appears linked with changes in environmental conditions, leading to breakdown of the plant antioxidant system as RLS symptoms generally develop concomitantly with leaf senescence (Schützendübel et al. 2008). For example, plants grown under high light conditions display higher disease levels than plants grown under normal light levels (Makepeace et al. 2008; McGrann and Brown 2018; Peraldi et al. 2014). Moreover, barley plants with altered redox homeostasis or delayed senescence also appear to show differential expression of RLS symptoms (McGrann et al. 2014, 2015a,b). The long latent period combined with the importance of seed transmission in R. collo-cygni dissemination (Havis et al. 2013) and the effects of abiotic stress on disease symptom formation has led to $R$. collo-cygni being described as an endophyte which can cause disease if it enters its necrotrophic stage (McGrann and Brown 2018; McGrann and Havis 2017). The exact causes of the transition from endophytic to necrotrophic lifestyle are currently unknown but the action of phytotoxic secondary metabolites (SM) has been proposed as a plausible mechanism (Heiser et al. 2003; Walters et al. 2008).

Many Dothideomycete fungi synthesize toxic SM during disease development (Stergiopoulos et al. 2013). SM such as the host-specific toxin victorin, produced by the Victoria blight pathogen of oat Bipolaris victoriae (syn. Cochliobolus victoriae), are required for pathogenicity (Lorang et al. 2004; Navarre and Wolpert 1999), whereas others such as the nonhost-specific phytotoxin dothistromin produced by Dothistroma septosporum, responsible for Dothistroma needle blight of pine trees, act as virulence factors mediating disease severity (Kabir et al. 2015). $R$. collo-cygni is known to produce light-activated phytotoxic SM called rubellins that have been isolated from $R$. collo-cygni-infected barley leaves (Miethbauer et al. 2003) and in vitro cultures (Heiser et al. 2004; Miethbauer et al. 
2006). Six rubellin derivatives, named alphabetically from A to E plus 14-dehydro rubellin $\mathrm{D}$, have been found in $R$. collo-cygni cultures (Miethbauer et al. 2008). Rubellins are nonhostspecific toxins that induce fatty acid peroxidation in vitro upon light activation (Heiser et al. 2004). Because light influences RLS symptom expression (Makepeace et al. 2008; Peraldi et al. 2014), the link between RLS and the action of rubellins in planta was presumed.

Most SM produced by plant-pathogenic fungi are derived from four core biosynthesis pathways that are controlled by polyketide synthases (PKS), nonribosomal peptide synthetases (NRPS), terpene cyclases (TC; synonym: terpene synthases), and dimethylallyl tryptophan synthases (DMATS) (MuriaGonzalez et al. 2015). Rubellin is an anthraquinone-derived toxin structurally related to characterized fungal SM such as cladofulvin, emodin, and aflatoxin, produced by Cladosporium fulvum, Aspergillus nidulans, and A. flavus, respectively. Based on the structural similarity between rubellin and other anthraquinone SM, rubellin biosynthesis has been proposed to occur through a polyketide pathway (Miethbauer et al. 2008). Considering that SM often mediate the interactions between a pathogen and its host, understanding the potential of $R$. collocygni for producing such compounds may provide insights into the nature of these interactions. Here, we investigated secondary metabolism in $R$. collo-cygni using the recently sequenced genome of this fungus (McGrann et al. 2016). We identified gene clusters involved in the synthesis of polyketide-derived
SM and demonstrated their transcriptional coregulation during $R$. collo-cygni colonization of the barley host and early stages of RLS symptom development.

\section{RESULTS}

\section{Identification of secondary metabolism-related} core genes in $\boldsymbol{R}$. collo-cygni.

Using Basic Local Alignment Search Tool (BLAST) combined with domain analysis, the $R$. collo-cygni genome was found to contain 21 genes encoding for proteins containing domains associated with PKS and 25 genes encoding proteins exhibiting a domain associated with NRPS. However, only 10 putative PKS possessed the three domains ketosynthase (KS), acyl-transferase (AT), and acyl carrier protein (ACP) required for prototypical PKS functionality. Of the ten putatively functional PKS, three exhibited the domain organization of nonreducing (NR)-PKS and seven had the organization of highly and partially reducing PKS (Fig. 1). A further three genes were classified as hybrid PKS-NRPS (HPS) genes because they encoded proteins containing a typical highly reducing PKS module fused to a single NRPS module consisting of condensation, adenylation, and peptidyl carrier protein domains (Fig. 1). The best BLAST hit for each R. collo-cygni PKS and HPS is given in Table 1. Furthermore, 10 NRPS appeared to have the domain organization found in functional type A and type B NRPS proteins. Three NRPS proteins were monomodular,

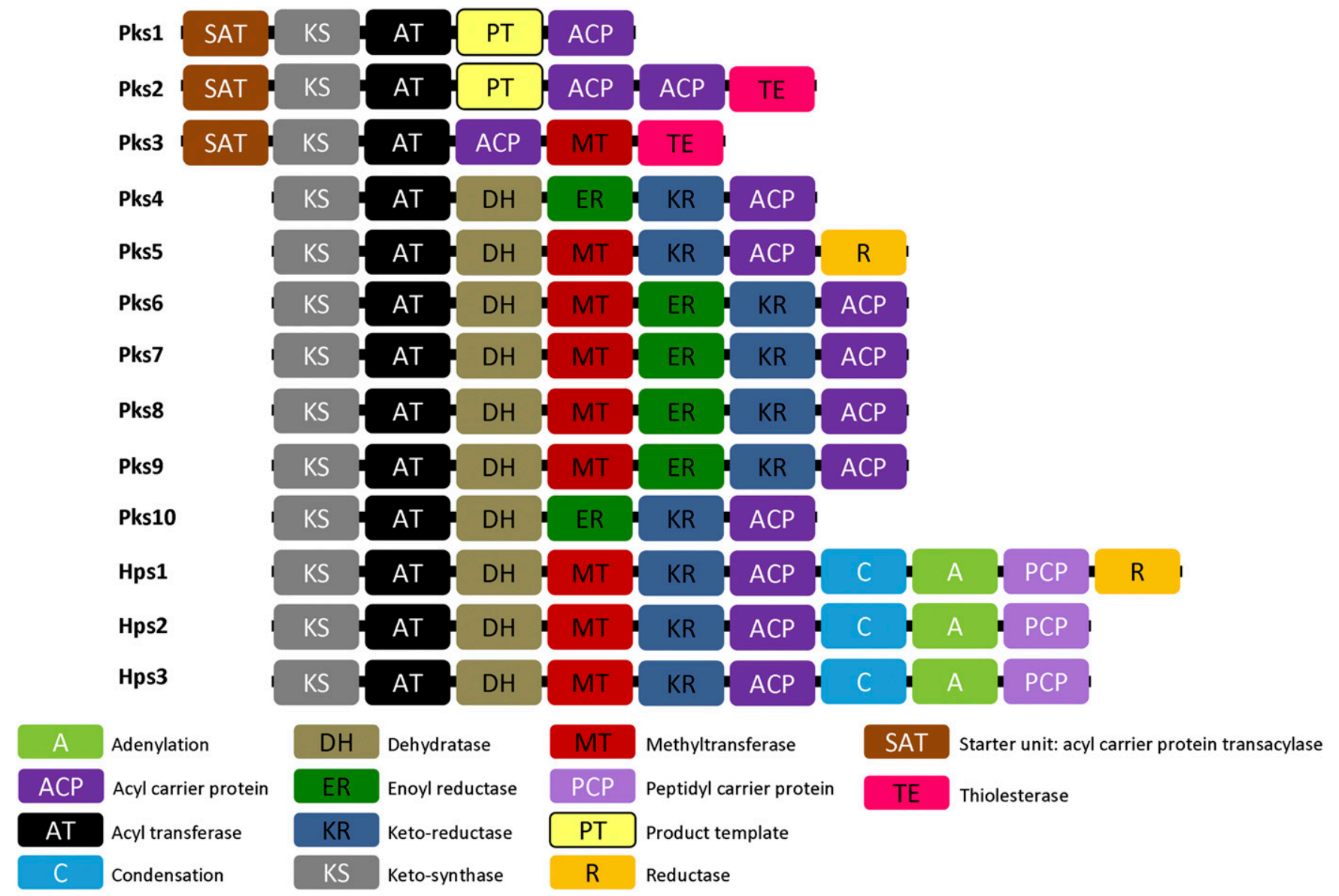

Fig. 1. Domain organization of the proteins encoded by Ramularia collo-cygni polyketide synthase and hybrid polyketide synthase/nonribosomal peptide synthetase (PKS-NRPS) genes. PKS and hybrid PKS-NRPS domains are labeled from N-terminus to C-terminus: starter unit acyl carrier transacylase (SAT), ketosynthase (KS), acyl transferase (AT), product template (PT), dehydratase (DH), methyl transferase (MT), enoyl reductase (ER), acyl carrier protein (ACP), condensation (C), adenylation (A), peptidyl carrier protein (PCP), thiolesterase (TE), and reductase (R). Only PKS-NRPS genes with domain structures found in functional secondary metabolite (SM) core proteins are shown. 
two were bimodular, and five were multimodular (Supplementary Fig. S1). Corresponding GenBank accession numbers for the $R$. collo-cygni SM core genes are presented in Supplementary Table S1. Compared with a previous analysis that identified only four TC and no DMATS in the $R$. collo-cygni genome (McGrann et al. 2016), it appears that $R$. collo-cygni possesses at least $27 \mathrm{SM}$ core genes. A similar number of SM core genes was obtained using the alternative detection methods Antibiotics and Secondary Metabolite Analysis Shell (AntiSMASH) and Secondary Metabolite Unique Regions Finder (SMURF) which identified 26 and 18 SM core genes, respectively (Supplementary Table S2). The number of core genes identified in $R$. collo-cygni is similar to that found in other closely related species of the class Dothideomycete such as Z. tritici, C. fulvum, and Pseudocercospora fijiensis (de Wit et al. 2012; McGrann et al. 2016; Ohm et al. 2012; Noar and Daub 2016). Thus, the $R$. collo-cygni genome encodes for an appropriate number of SM core genes but these do not appear to reflect completely the lifestyle of the fungus because no correlation was apparent between fungi with different lifestyles in the order Capnodiales (Supplementary Table S3).

\section{Prediction of $R$. collo-cygni PKS and HPS secondary metabolism gene clusters.}

The genomic locus around each putatively functional PKS and HPS gene was investigated by in silico genome walking. Eight of the identified PKS and all three HPS core genes appeared to be located in a cluster containing several genes with a predicted function associated with secondary metabolism (Fig. 2). $P k s 8$ and $P k s 2$ were not located within potential SM gene clusters but these PKS sequences were located at an end of their respective contig or scaffold; thus, it is possible that SM-related genes are present near these core genes but these regions were not completely assembled in the draft genome assembly. The largest cluster, located on contig 58 near Pks6, was over $45.2 \mathrm{~kb}$ in length and contained 16 putative SM-related genes and two predicted genes which currently have no transcript evidence based on RNAseq data (McGrann et al. 2016). The smallest cluster spanned $17 \mathrm{~kb}$ on contig 282 and contained five SM-related genes, including the PKS core gene $(P k s 10)$ and a TC gene (Fig. 2, gene Tcl) previously identified in the R. collo-cygni genome (McGrann et al. 2016). Of the eight putative SM clusters located around PKS, four appeared to have more than one predicted core gene present within the cluster. The cluster on contig 118 contained two genes with predicted PKS domains; however, only Pks3 exhibited the typical domain organization of functional PKS. Two other clusters, located on contig 280 and contig 58 near $P k s 9$ and $P k s 6$, both contained an additional SM gene exhibiting a domain architecture found in nonfunctional NRPS. These genes were designated Nps12 (contig 280) and Nps13 (contig 58). Of the 11 clusters surrounding PKS or HPS cores genes, six possessed at least one gene with a predicted function associated with cellular transport from either the major facilitator superfamily (MFS) or ATP-binding cassette family, which are known to be involved in secondary metabolism processes, including protecting fungi against their own toxic SM (Gardiner et al. 2005). Two putative SM clusters surrounding a PKS core gene ( $P k s 1$ and Pks6, identified on scaffold $\mathrm{m} 24$ and contig 58, respectively) and two located near a HPS gene (Hps 1 and Hps2, identified on contig 1 and contig 14, respectively) contained predicted transcription factors with GAL4 domains, also known as $\mathrm{Zn}(\mathrm{II})_{2} \mathrm{Cys}_{6}$ DNA-binding domains, that have been shown to regulate secondary metabolism in fungal pathogens (Brakhage 2013).

Three of the SM-related gene clusters found in R. collo-cygni exhibited high similarity to clusters involved in the biosynthesis of SM previously characterized in another fungal species (Fig. 3; Supplementary Table S4). Four of the six genes identified in the cluster located on contig 30 containing Pks5 are similar to genes present in the betaenone biosynthesis cluster identified in the oilseed rape pathogen Phoma betae (syn. Pleospora betae) (Ugai et al. 2015) (Fig. 3A). Pksl was located in a cluster exhibiting similarity to an SM-cluster involved in monodictyphenone production in A. nidulans (Chiang et al. 2010) (Fig. 3B). Similarly, the locus on contig 14 surrounding Hps 2 comprises genes showing similarity to all seven genes present in the chaetoglobosin A biosynthetic cluster identified in Penicillium expansum as well as two genes not identified in the P. expansum cluster (Schümann and Hertweck 2007) (Fig. 3C). The clusters identified on contigs $282,118,58,1$, and 38 , surrounding $P k s 10, P k s 3$, Pks6, Hps 1, and Hps3, respectively, all showed similarity to SM clusters present in other fungal species but the end SM products of these clusters are currently unknown (Supplementary Fig. S2). Because all of the $R$. collo-cygni clusters that showed similarity to SM gene clusters in other fungi typically exhibited gene inversions and rearrangements within the $R$. collo-cygni locus, it remains to be determined whether or not $R$. collo-cygni can synthesize these SM products.

The relationship between $R$. collo-cygni core genes and core genes in other fungal species was further examined by a

Table 1. Ramularia collo-cygni secondary metabolite core polyketide synthase (PKS) and hybrid PKS-nonribosomal peptide synthase (NRPS) gene complement

\begin{tabular}{|c|c|c|c|c|c|c|}
\hline $\begin{array}{l}\text { R. collo-cygni } \\
\text { gene }\end{array}$ & Best BLAST hit ${ }^{\text {a }}$ & $\begin{array}{c}\text { Fungal } \\
\text { organism }\end{array}$ & $\begin{array}{c}\text { Accession } \\
\text { number }\end{array}$ & $e$-value & $\begin{array}{c}\text { Amino acid } \\
\text { identity }(\%)\end{array}$ & $\begin{array}{c}\text { Potential } \\
\text { product }\end{array}$ \\
\hline Pks 1 & PKS & Umbilicaria pustulata & SLM39055.1 & 0.0 & 67 & Rubellin $^{\mathrm{b}}$ or uredinorubellin ${ }^{\mathrm{c}}$ \\
\hline$P k s 2$ & PKS & Zymoseptoria tritici & XP_003848644.1 & 0.0 & 83 & $\ldots$ \\
\hline$P k s 3$ & PKS & Z. tritici & XP_003855816.1 & 0.0 & 71 & $\ldots$ \\
\hline Pks4 & Hypothetical & Podospora anserina & XP_001904114.1 & 0.0 & 49 & $\ldots$ \\
\hline$P k s 5$ & PKS & Z. tritici & XP_003856913.1 & 0.0 & 68 & Betaenone $^{\mathrm{d}}$ \\
\hline Pks6 & Putative PKS & Z. tritici & XP_003855801.1 & 0.0 & 56 & $\ldots$ \\
\hline Pks7 & LovF & Tolypocladium ophioglossoides & KND92549.1 & 0.0 & 33 & $\ldots$ \\
\hline Pks 8 & PKS & Cenococcum geophilum & OCK92294.1 & 0.0 & 60 & $\ldots$ \\
\hline$P k s 9$ & PKS like & Z. brevis & KJX93771.1 & 0.0 & 80 & $\ldots$ \\
\hline Pks 10 & Putative PKS & Neofusicoccum parvum & XP_007581490.1 & 0.0 & 59 & $\ldots$ \\
\hline Hps 1 & PKS-NRPS & Cercospora beticola & PIB02931.1 & 0.0 & 71 & $\ldots$ \\
\hline Hps 2 & Putative PKS-NRPS protein & Penicillium expansum & CAO91861.1 & 0.0 & 59 & Chaetoglobosin $^{\mathrm{d}}$ \\
\hline Hps3 & Hypothetical & Polytolypa hystricis & PGH21386.1 & 0.0 & 69 & $\ldots$ \\
\hline
\end{tabular}

${ }^{\text {a }}$ Hypothetical $=$ hypothetical protein and LovF $=$ lovastatin diketide synthase.

b Reported by Miethbauer et al. (2003).

c Reported by Miethbauer et al. (2008).

${ }^{\mathrm{d}}$ Potential product reported in this study based on gene synteny of the $R$. collo-cygni biosynthetic cluster. 
phylogenetic analysis of the protein sequences encoded by these genes (Supplementary Fig. S3). Among the NR-PKS, the protein encoded by $P k s l$ appeared to be closely related to that encoded by Acas, ClaG, EncA, and $M d p G$, involved in atrochrysone carboxylic acid, cladofulvin, endocrocin, and monodictyphenone biosynthesis in A. terreus, C. fulvum, A. fumigatus, and $A$. nidulans, respectively (Awakawa et al. 2009; Chiang et al. 2010; Griffiths et al. 2016; Lim et al. 2012). The HPS encoded by Hps 2 was located on the same clade as the enzyme encoded by CheA, the core gene involved in the chaetoglobosin A biosynthesis pathway in $P$. expansum (Schümann and Hertweck 2007). Chaetoglobosins are alkaloid cytochalasans that contain an isoindoline backbone fused to a macrocyclic ring. HPS involved in cytochalasan synthesis are known to utilize a specific range of amino acids. Chaetoglobosins are synthesized from tryptophan, cytochalasins from phenylalanine, alachalasins from alanine, scoparasins from tyrosine, and aspochalasins from leucine (Scherlach et al. 2010). AntiSMASH-based predictions showed that the A-domain in Hps2 is likely to incorporate leucine, tryptophan, or alanine, suggesting that the product of Hps 2 could be a cytochalasan, potentially belonging to the chaetoglobosin, aspochalasin, or alachalasin family (data not shown). The in silico analysis of the $R$. collo-cygni genome demonstrates that $R$. collo-cygni possesses genetic loci that may be able to synthesize a wide range of SM.

\section{Gene expression profiling of $\boldsymbol{R}$. collo-cygni SM-related} genes during RLS development.

Changes in transcript levels of $R$. collo-cygni PKS and HPS SM core genes were investigated during $R$. collo-cygni colonization and RLS development in artificially inoculated barley seedlings using quantitative reverse-transcription polymerase chain reaction (qRT-PCR). Primers with amplification efficiency suitable for qRT-PCR were successfully designed for six
PKS (Pks5, Pks4, Pks10,Pks3,Pks2, and Pks1) and all three HPS.

RLS symptoms developed significantly over time $(P<$ 0.001 ), with small pepper spots typical of the earliest stages of the disease first visible from 7 to 10 days postinoculation (dpi) onward (Fig. 4A). Subsequently, lesions expanded to form larger spots which eventually coalesced to cover larger portions of the leaf from 15 to 21 dpi onward. As RLS symptoms developed, green leaf area (GLA) retention significantly declined over time $(P<0.001$; Fig. 4B). Loss of GLA was slower between 5 and 7 dpi $(P<0.05)$ but rapidly increased from $10 \mathrm{dpi}$ onward $(P<0.001)$. R. collo-cygni biomass assessed as accumulation of fungal DNA also increased significantly during the inoculation time course $(P=0.001$; Fig. 4C). However, significant increases between consecutive time points were only noted between 15 and 18 dpi $(P=0.019)$, with the amount of fungal DNA plateauing at 18 and $21 \mathrm{dpi}$. Significant changes in $R$. collo-cygni colonization stages were also observed over time. The number of stomata penetrated by fungal hyphae and subsequently developing conidiophore-like structures gradually increased over the duration of the time course $(P<0.001$; Supplementary Fig. S4). Necrosis associated with stomata significantly increased from $10 \mathrm{dpi}$ onward $(P=0.006)$.

Transcripts for five of the six PKS tested were most abundant at 5 and $7 \mathrm{dpi}$, corresponding to the asymptomatic and the early symptom formation stage, respectively. Expression of $P k s 5$, $P k s 4, P k s 2$, and $P k s 1$ was highest at $5 \mathrm{dpi}$, with transcript levels subsequently declining after $10 \mathrm{dpi}$. PkslO transcript levels were highest at both 5 and $7 \mathrm{dpi}$ but decreased rapidly at $10 \mathrm{dpi}$ and remained low thereafter (Fig. 5). In contrast, the NR-PKS, $P k s 3$, showed consistently low transcript levels that did not change over the infection time course. Transcript levels of all three HPS were most abundant during the early symptom development phase, with the expression of Hps 1 and Hps3

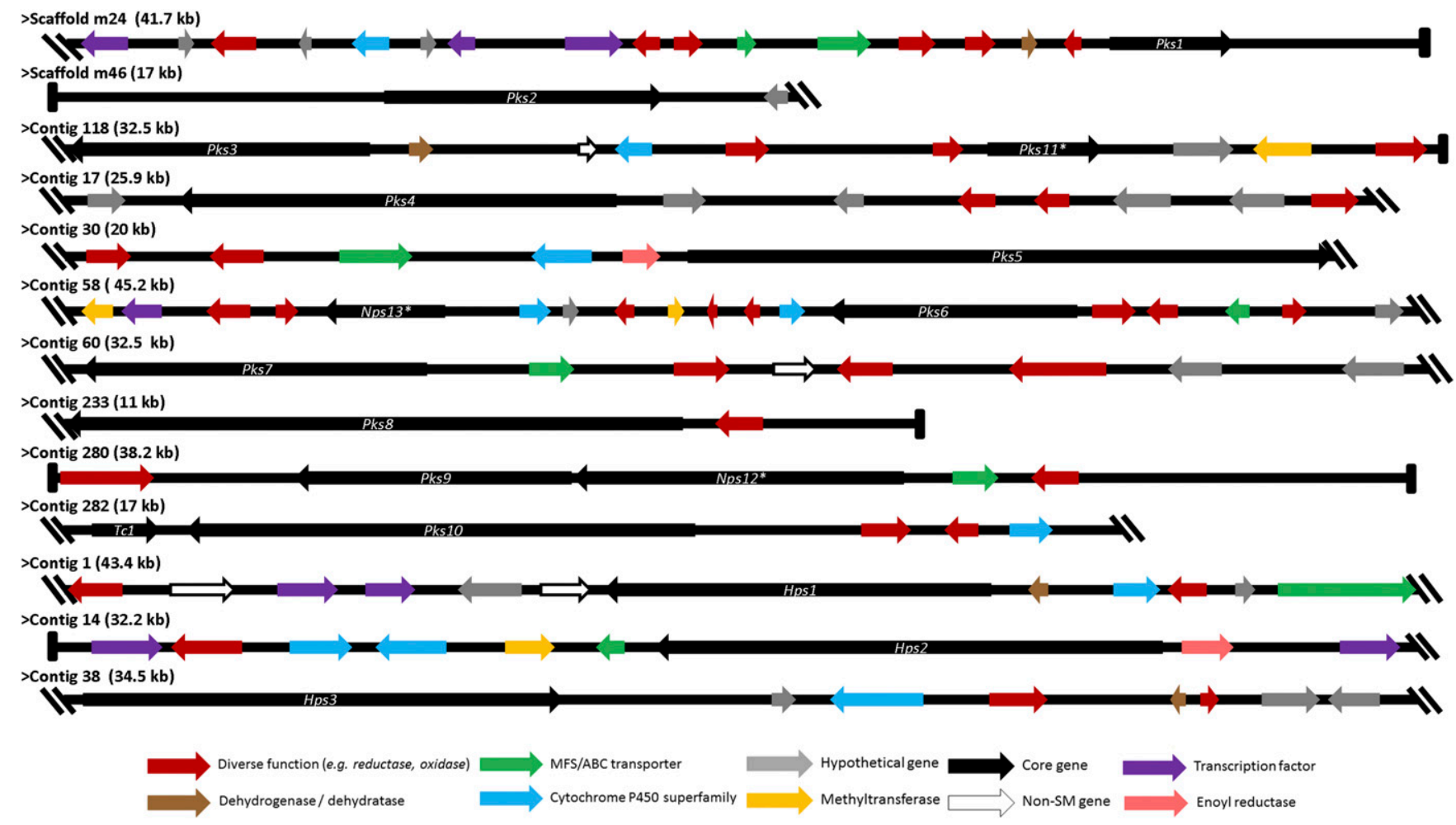

Fig. 2. Organization of the genetic loci around Ramularia collo-cygni secondary metabolism core genes. Arrowheads indicate predicted direction of transcription for each open reading frame. The end of a cluster is marked by a double oblique bar and the end of a contig or scaffold is marked by a single vertical bar. Brackets indicate the size of the cluster. Each cluster is drawn to scale. Secondary metabolite core genes with domain structures that would indicate the resultant protein is nonfunctional are marked with an asterisk. 
peaking at $7 \mathrm{dpi}$ and slowly decreasing to minimal levels at 21 dpi, whereas Hps 2 was highly expressed at both 5 and 7 dpi before declining to a low level of expression from 10 dpi onward (Fig. 5). The expression profile of several NRPS indicated that these SM core genes were also most abundant at 5 and 7 dpi (Supplementary Fig. S5). SM core gene transcription was highest when foliar $R$. collo-cygni biomass was at its lowest. Concomitant with the expression of RLS symptoms, there is a decline in the host's antioxidant system (Schützendübel et al. 2008) which is associated with elevated levels of reactive oxygen species hydrogen peroxide in the plant (McGrann and Brown 2018). Therefore, $R$. collo-cygni catalase expression was measured over the time course of disease development as an experimental control gene that would be predicted to increase transcript abundance as disease developed, similar to the interaction between the related fungus $Z$. tritici and wheat (Keon et al. 2005). As $R$. collo-cygni biomass increased over the experiment time course along with RLS symptom expression, transcript levels of $R$. collo-cygni catalase, a hydrogen peroxide scavenger, also increased (Fig. 5). These results suggest that elevated $R$. collo-cygni SM core gene expression was distinct from fungal biomass accumulation and preceded by or overlapped with early disease symptom expression.

Because the expression profile of most of the $R$. collo-cygni SM core genes investigated here showed similar patterns of transcription, the expression of previously characterized fungal transcriptional regulators as well as SM pathway-specific transcription factors (TF) was investigated to determine possible coregulation of these genes. Putative $R$. collo-cygni homologs of the zinc-finger TF PacC, CreA, and AreA, involved in responses to $\mathrm{pH}$, carbon, and nitrogen conditions, respectively; VeA and LaeA, two components of the lightactivated velvet complex which regulates diverse biological processes, including asexual reproduction and secondary metabolism; and pathway-specific TF $A f l R$ and $A f l J$, both known to regulate genes involved in the aflatoxin and dothistromin biosynthetic cluster in A. flavus and D. septosporum, were identified in the $R$. collo-cygni genome using BLASTp (Supplementary Table S5). Transcript profiles of PacC and AreA were similar to those observed for most of the SM core genes, with highest transcript levels at 5 and 7 dpi before a general decline in gene expression from 10 dpi onward (Fig. 6A). The pathway-specific TF $A f l R$ and $A f l J$ were also most highly expressed during the early stages of $R$. collo-cygni colonization (Fig. 6C). Both $V e A$ and $L a e A$ exhibited slightly different expression profiles, with the highest transcription of these genes between 5 and $10 \mathrm{dpi}$ (Fig. 6B). These results indicate that several transcription regulators with known roles in SM regulation in plant-pathogenic fungi appear coregulated with most of the $R$. collo-cygni SM core genes examined. The increased expression of SM core genes during the early stages of $R$. collocygni colonization suggests that, if these SM are indeed produced by $R$. collo-cygni, the SM products of the genes investigated here could play a role in early plant-pathogen interactions such as manipulating the host to aid fungal colonization or to protect the fungus from competing organisms.

In vitro antifungal activity of $R$. collo-cygni.

To assess antifungal activity of SM produced by $R$. collocygni, in vitro dual-culture competition assays between $R$. collocygni and common fungal pathogens of barley were used. The presence of $R$. collo-cygni significantly inhibited the growth of all of the tested competing fungi examined (Fig. 7). Growth of Pyrenophora teres, the causative agent of net blotch, was inhibited by $66 \%(P=0.041)$ and $80 \%(P=0.002)$ on alkyl ester agar (AEA) and potato dextrose agar (PDA) media,

A



B

Rcc (unknown SM)

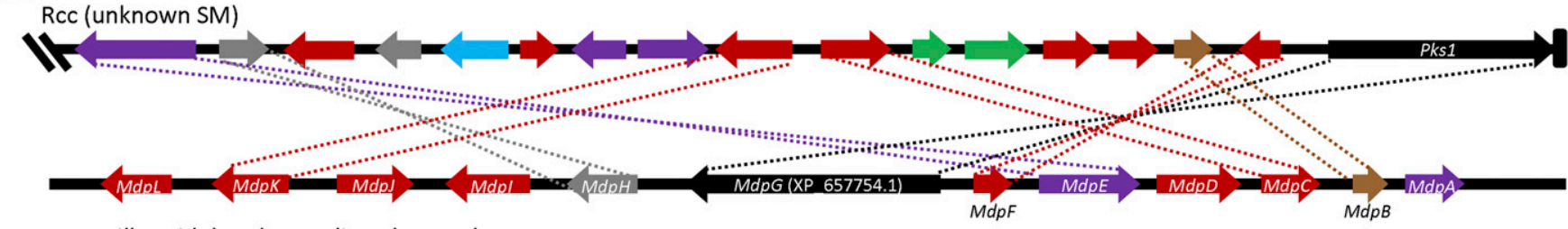

Aspergillus nidulans (monodictyphenone)

C

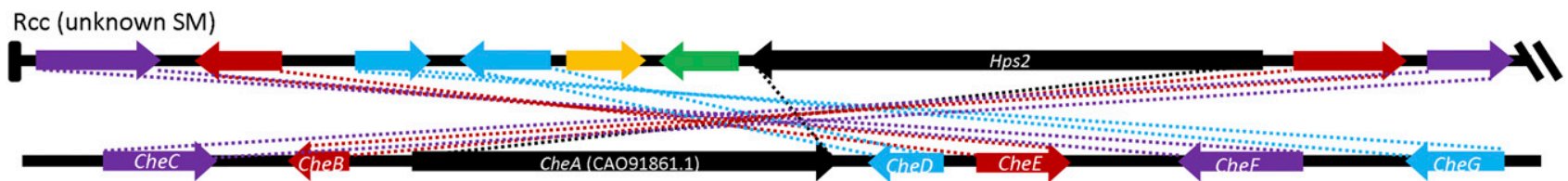

Penicillium expansum (chaetoglobosin A)

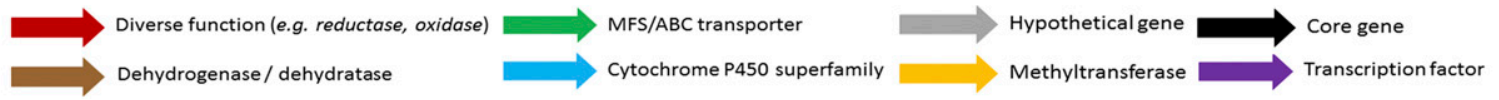

Fig. 3. Synteny between selected Ramularia collo-cygni secondary metabolism clusters and gene clusters in other fungi. Syntenic relationship between predicted $R$. collo-cygni secondary metabolism clusters and gene clusters from A, Phoma betae; B, Aspergillus nidulans; and C, Penicillium expansum are shown. Arrowheads indicate open reading frame predicted transcription direction. Putative orthologous genes identified by reciprocal BLAST are marked by dotted lines. The end of a cluster is marked by a double oblique bar and the end of a contig or scaffold is marked by a single vertical bar. 
respectively. Fusarium poae growth was inhibited on both media by more than $50 \%$ in the presence of $R$. collo-cygni (AEA, $P<0.001$; PDA, $P<0.001)$ and that of Fusarium graminearum by more than $60 \%$ (AEA, $P<0.001$; PDA, $P<$ 0.001). R. collo-cygni also had an antagonistic effect on Rhynchosporium commune, responsible for barley leaf scald, growth $(P<0.001)$ on PDA. A red coloration of the medium surrounding $R$. collo-cygni cultures that was particularly visible on PDA when grown in competition with P. teres and Rhynchosporium commune (Fig. 7B) suggests that $R$. collo-cygni releases compounds into the growing medium that inhibits the growth of other fungi. Previous reports have demonstrated that the one characterized SM family synthesized by $R$. collo-cygni, the rubellins, can produce a red coloration in culture media (Heiser et al. 2003). Together, these data illustrate that SM could provide $R$. collo-cygni with a competitive advantage over other microorganisms during host colonization.

\section{DISCUSSION}

Genomic analysis of the $R$. collo-cygni genome reveals the potential to produce several different SM.

Genomes of filamentous species of Ascomycetes and, in particular, of Dothideomycetes possess a large number of SM- related genes which result in the ability to produce a wide range of chemical compounds (Bushley and Turgeon 2010; Kroken et al. 2003). The $R$. collo-cygni genome contains at least 23 potentially functional SM core genes belonging to the PKS, NRPS, and HPS families. This finding contrasts with results of McGrann et al. (2016), which reported 19 PKS, 14 NRPS, and $4 \mathrm{TC}$ but did not report any dimethylallyl tryptophan synthases or HPS. This variation highlights the importance of analyzing domain structure rather than relying on BLAST similarity alone to identify SM core genes, which can lead to misidentification of putatively nonfunctional genes as well as excluding HPS. Core genes tend to be organized within the $R$. collo-cygni genome in SM-related gene clusters. Clusters exhibiting similarity with previously reported fungal SM clusters involved in the biosynthesis of characterized SM were identified in the $R$. collo-cygni genome (Fig. 3). The cluster located at the Pks5 loci contained putative homologs to all the genes involved in betaenone biosynthesis in Phoma betae (Ugai et al. 2015). Betaenones are phytotoxins associated with leaf spot disease of sugar beet and induce leaf chlorosis (Ichihara et al. 1983). The cluster located near the core gene Hps 2 contained putative homologs of the seven genes involved in the biosynthesis of chaetoglobosin A in Penicillium expansum and two additional genes, a putative O-methyltransferase and an MFS transporter.



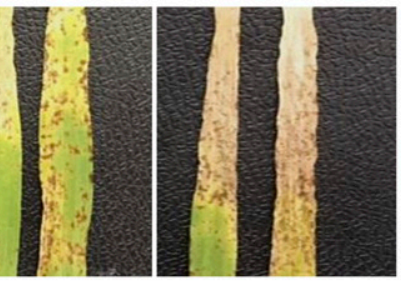

$12 \mathrm{dpi}$

C

$15 \mathrm{dpi}$

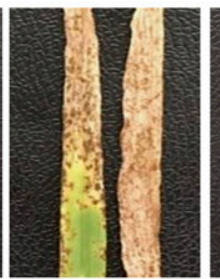

$18 \mathrm{dpi}$

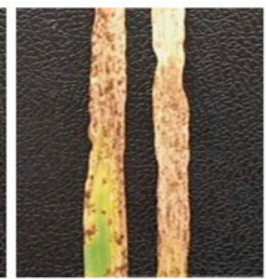

$21 \mathrm{dpi}$

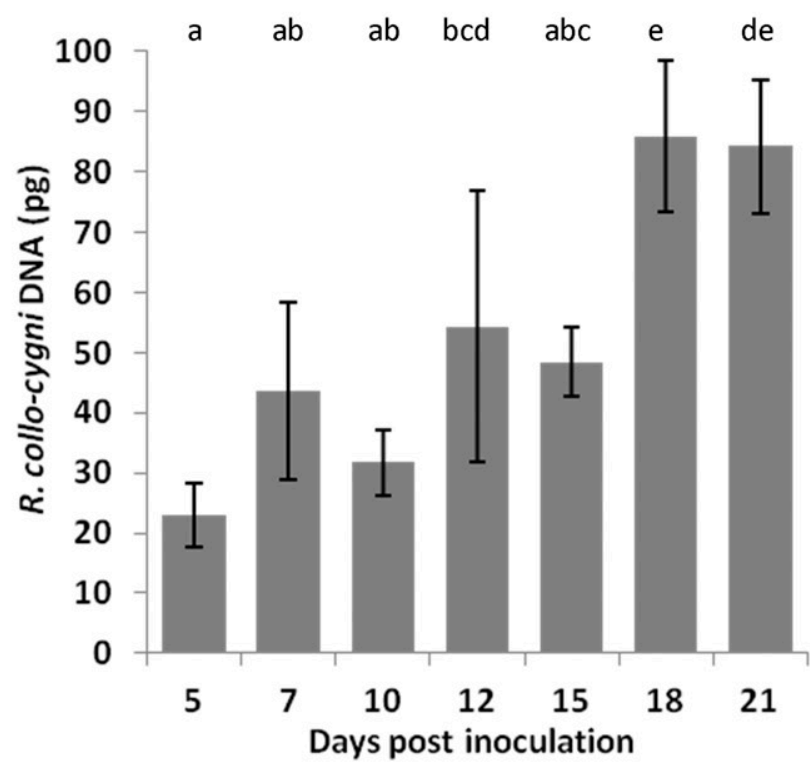

Fig. 4. Development of Ramularia leaf spot (RLS) in barley Century seedlings. A, Representative images of RLS symptoms on barley seedlings over a 21-day time course following inoculation with Ramularia collo-cygni DK05 Rcc001 ss2; dpi = days postinoculation B, Decline in green leaf area (GLA) retention (black line) and increase in RLS symptom development (gray bars) measured over the 21-day inoculation time course as the area under the disease progress curve (AUDPC). AUDPC is presented as a proportion of the maximum AUDPC possible over the entire inoculation course. Time points that showed significantly different development of RLS symptoms (top row) or differences in GLA retention (bottom row) from each other $(P<0.05)$ are indicated by different letters. C, $R$. collo-cygni DNA levels (in picograms) in barley prophyll leaves measured by quantitative polymerase chain reaction. Fungal DNA levels (in picograms) were calculated using a standard curve produced from a dilution series of $R$. collo-cygni DNA. Time points that showed significantly different $(P<0.05)$ amounts of fungal DNA are indicated by different letters. 
Chaetoglobosins are known to have cytotoxic properties (Ohtsubo et al. 1978) and to exhibit antifungal activity (Zhang et al. 2013). Therefore, although, to date, only rubellins were isolated from $R$. collo-cygni, it appears that $R$. collo-cygni has the genetic capacity to produce a wide range of SM, including several phytotoxins and antifungal agents. $R$. collo-cygni gene clusters with similarity to uncharacterized SM biosynthetic clusters, where the function of the SM has not yet been determined in other species, were also discovered, further highlighting the potential diversity of the R. collo-cygni SM arsenal.

The pathway responsible for rubellin biosynthesis has yet to be determined but the chemical structure of this SM may
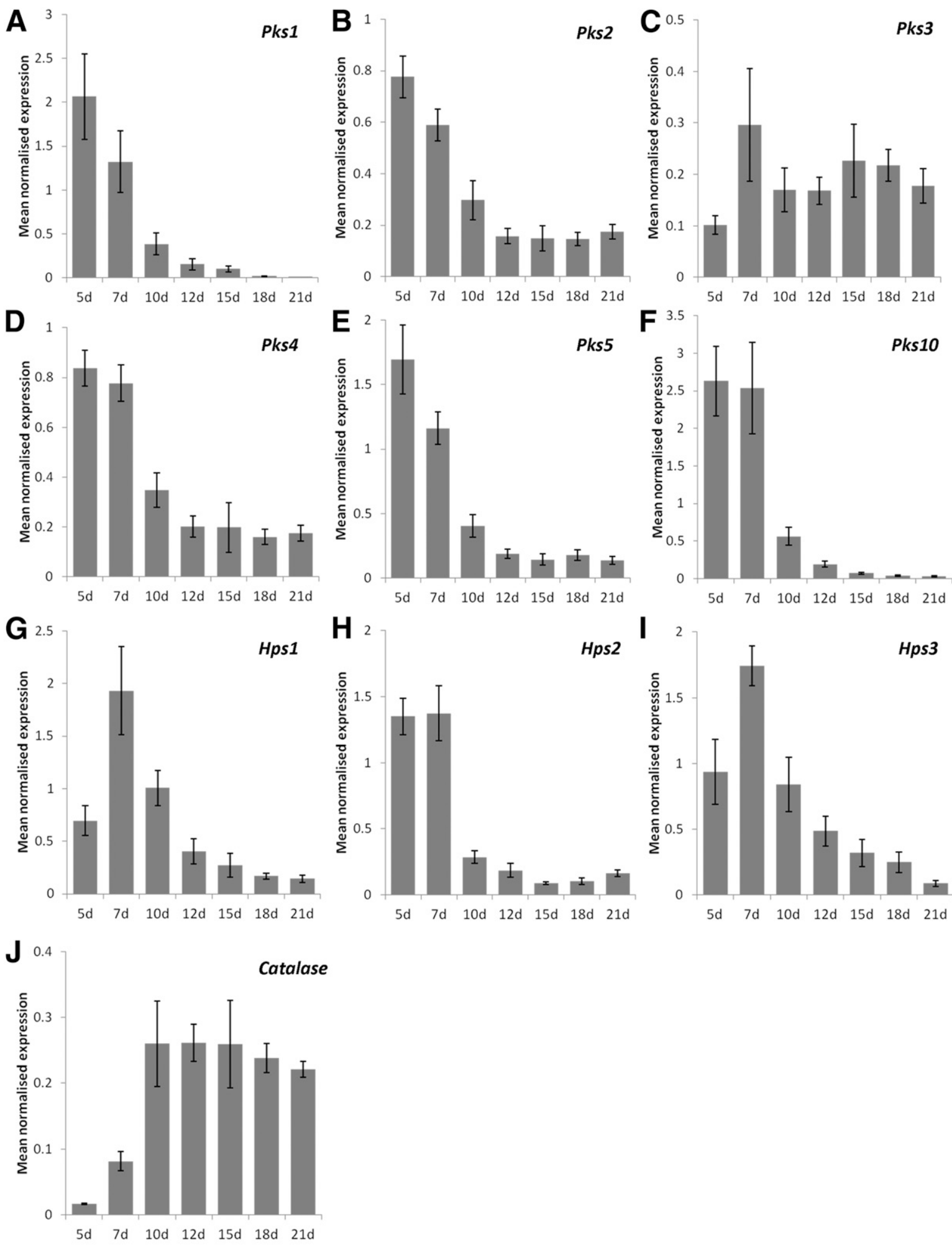

Fig. 5. Expression of selected Ramularia collo-cygni polyketide synthases (PKS) and hybrid polyketide synthase/nonribosomal peptide synthetase (HPS) genes during Ramularia leaf spot development in barley seedlings. A, Pks1; B, Pks2; C, Pks3; D, Pks4; E, Pks5; F, Pks10; G, Hps1; H, Hps2; I, Hps3; and J, Catalase. Data are presented as mean normalized expression values \pm standard error over a 21-day time course postinoculation. The expression of the $R$. collocygni catalase gene is shown as a control. 
provide clues toward better understanding its production. Chemically, rubellins resemble several fungal polyketidederived SM such as endocrocin, emodin, and cladofulvin that all exhibit a dioxoanthracene backbone characteristic of anthraquinones and are synthesized by the NR-PKS EncA, MdpG, and $C l a G$, in A. fumigatus, A. nidulans, and C. fulvum, respectively (Chiang et al. 2010; Griffiths et al. 2016; Lim et al. 2012). Three NR-PKS ( $P k s 3, P k s 1$, and $P k s 2)$ were identified in the $R$. collo-cygni genome. Of these $R$. collo-cygni NR-PKS, $P k s 3$ can probably be excluded from the biosynthesis of rubellin or other anthraquinone-derived metabolites because the domain organization of $P k s 3$, which includes a methyltransferase domain and lacks a product template domain, differs significantly from the SAT-KS-AT-PT-ACP organization of $E n c A, M d p G$, and $C l a G$. Furthermore, the phylogenetic analysis suggests that the protein encoded by $P k s 3$ is not related to proteins involved in anthraquinone biosynthesis. The domain organization of $P k s 2$ is similar to that of other NR-PKS, including $A f P k s A$ and $D s P k s A$, involved in the production of the anthraquinone-derived $A$. flavus aflatoxin and $D$. septosporum dothistromin, as well as the PKS encoded by CTB1, Pks1, and Cppks1 involved in the biosynthesis of the perylenequinone toxins cercosporin, elsinochrome, and phleichrome, isolated from Cercospora nicotianae, Elsinoë fawcettii, and Cladosporium phlei, respectively (Choquer et al. 2005; Liao and Chung 2008; So et al. 2015). Of these NR-PKS, the phylogenetic
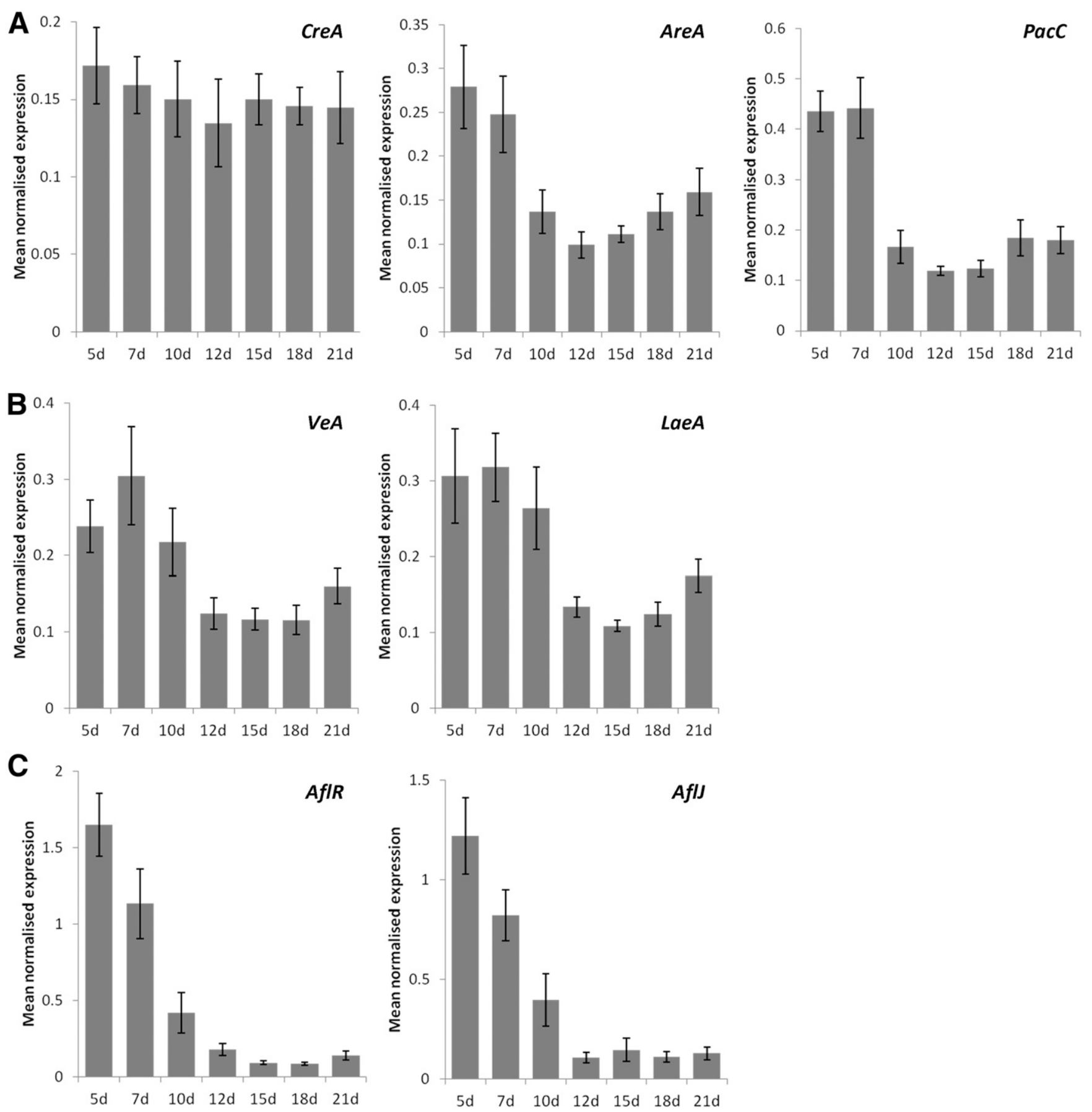

Fig. 6. Expression of putative Ramularia collo-cygni homologs of characterized fungal secondary metabolism regulators during Ramularia leaf spot development in barley seedlings. A, Transcriptional regulators PacC, CreA, and AreA; B, components of the velvet complex LaeA and VeA; and $\mathbf{C}$, transcription factors AflR and AflJ. Data are presented as mean normalized expression values \pm standard error over a 21-day time course postinoculation. 
analysis suggests that $P k s 2$ is more closely related to the elsinochrome synthase $P k s 1$ and the phleichrome synthase Cppksl than AfPksA and DsPksA, which are recovered in a different clade. These results suggest that $P k s 2$ may be involved in either the production of an anthraquinone-derived SM or the biosynthesis of SM potentially belonging to the perylenequinone family.

$P k s 1$ encodes a protein exhibiting the same domain organization as the proteins encoded by $M d p G, E n c A$, and $C l a G$. R. collo-cygni PKS Pksl was also closely related to $M d p G$,
EncA, and ClaG, as well as Acas, which is responsible for the biosynthesis of atrochrysone carboxylic acid, a precursor of endocrocin, emodin, chrysophanol, and cladofulvin (Awakawa et al. 2009; Griffiths et al. 2016). A putative role for Pksl in emodin or chrysophanol biosynthesis was further supported by the colocalization of $P k s l$ with genes exhibiting sequence similarity to those identified in the monodictyphenone biosynthetic cluster involved in emodin and chrysophanol production (Fig. 3B) (Chiang et al. 2010). Considering that the rubellins reported from $R$. collo-cygni, especially rubellin A

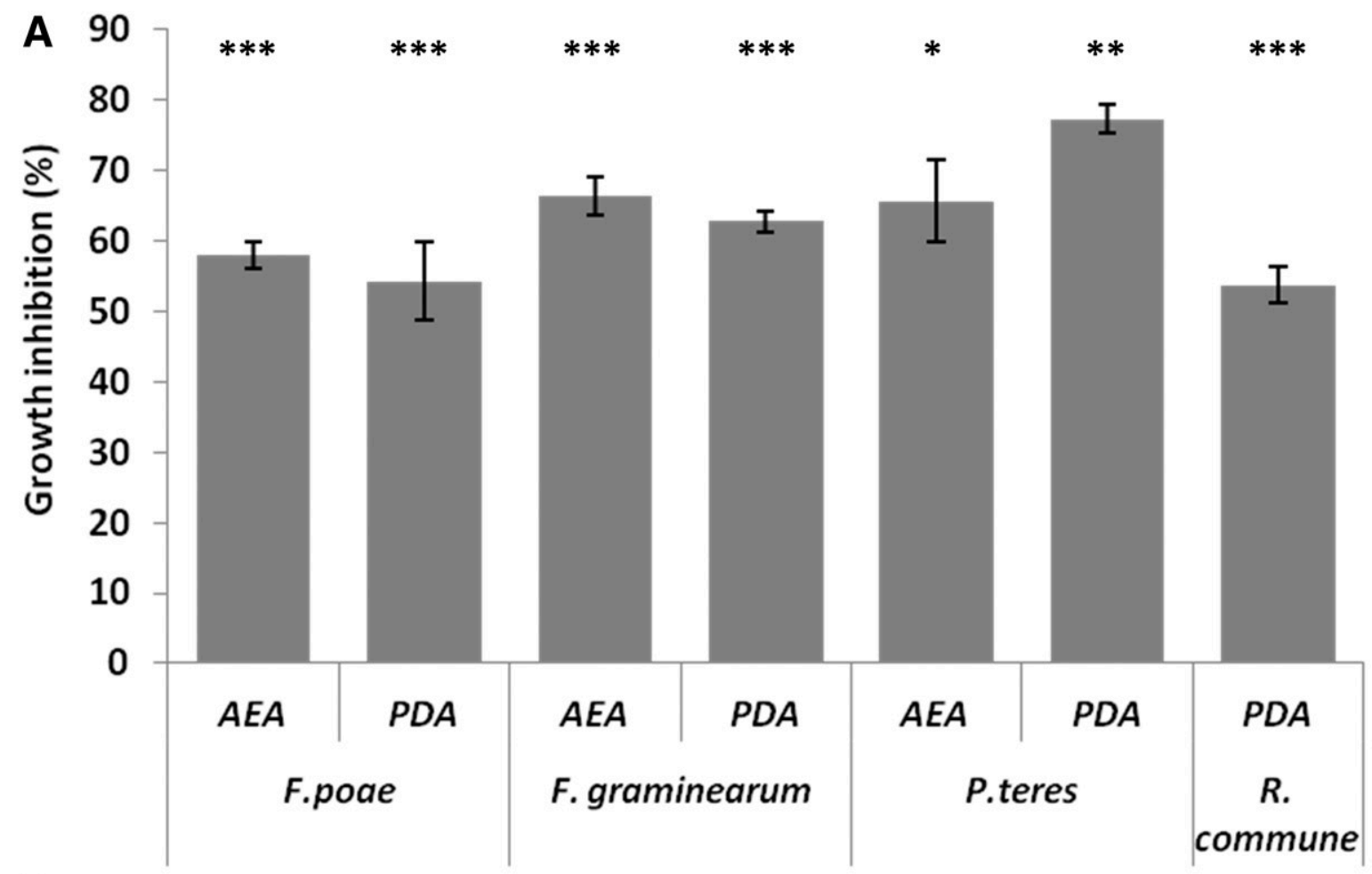

\section{B i)}

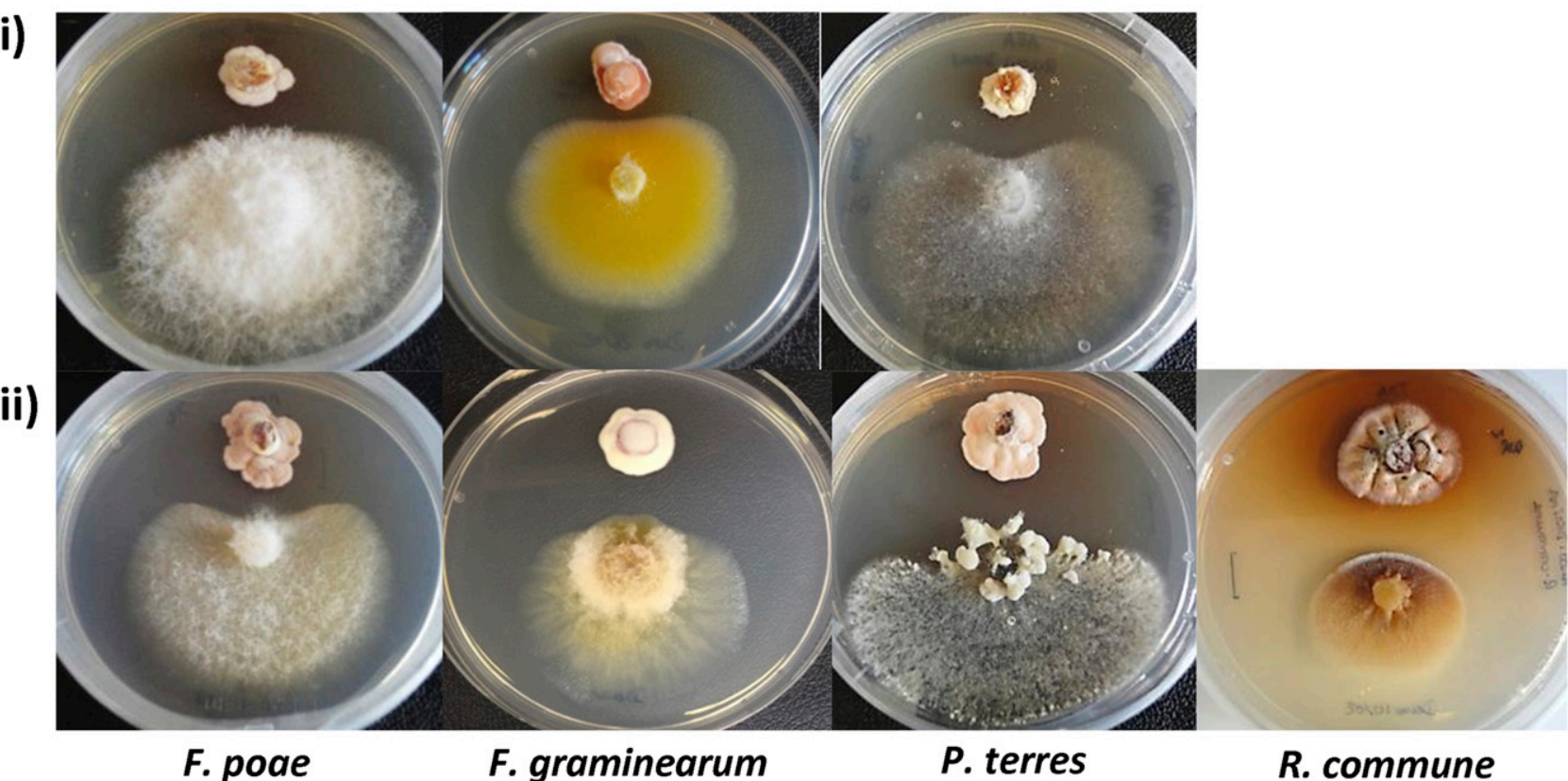

Fig. 7. Growth inhibition assays between Ramularia collo-cygni and common foliar fungal pathogens of barley. A, Growth inhibition of fungal pathogens in the presence of $R$. collo-cygni. Data are presented as the average growth inhibition (percentage) of each fungal pathogen \pm standard error. Asterisks $* * *, * *$, and $*$ indicate $P<0.001,0.01$, and 0.05 , respectively. B, Representative images of the dual plate assay between $R$. collo-cygni (placed in the top position in each plate) and Fusarium poae, F. graminearum, Pyrenophora teres, and Rhynchosporium commune (bottom position) after 10, 7 , 10, and 21 days, respectively, when grown on (i) alkyl ester agar (AEA) and (ii) potato dextrose agar (PDA). 
and B, and uredinorubellins (Miethbauer et al. 2008) exhibit a chemical structure resembling that of a dimer of chrysophanol, $P k s l$ appears to be a strong PKS candidate involved in the rubellin biosynthetic pathway. Functional characterization of this gene and its genetic locus may provide further insights into the pathways that regulate rubellin biosynthesis.

\section{$R$. collo-cygni secondary metabolism core gene transcripts are most abundant during the early stages of $\boldsymbol{R}$. collo-cygni colonization.}

Transcript levels of core genes associated with potential secondary metabolism pathways in $R$. collo-cygni were most abundant during the asymptomatic and the earliest lesion formation phases following $R$. collo-cygni colonization (Fig. 5). Transcription of SM-related genes in Colletotrichum orbiculare, C. higginsianum, and C. gloeosporioides were most abundant during the early stages of infection (Alkan et al. 2015; Gan et al. 2013; O'Connell et al. 2012). In the R. collo-cygni sister species Z. tritici, SM gene transcripts were most abundant during the transition phase from biotrophic to necrotrophic growth (Rudd et al. 2015). SM produced during this transition phase could be involved in protecting the fungus from plant defense mechanisms or inducing host cell death to facilitate fungal development during necrotrophic colonization. However, some secondary metabolism-associated transcripts were found to be most abundant during later stages of disease development in Z. tritici, including sporulation (Cairns and Meyer 2017; Palma-Guerrero et al. 2016). Similarly, in the closely related $D$. septosporum, four core genes, including three PKS, were upregulated during the later stages of infection (Bradshaw et al. 2016). The variation in SM transcription profiles between different species of Dothideomycetes highlights the complex nature of SM functions in these fungi and may provide insights into how SM affects the unique pathology of the associated diseases.

Regulation of fungal SM-related genes is the result of a finely tuned balance between requirements for fungal development and secondary metabolism (Calvo et al. 2002). As a result, SMrelated genes are often coregulated, facilitating rapid control of SM production. $R$. collo-cygni SM core gene expression appeared to be coregulated with several well-characterized fungal transcriptional regulators, including the $\mathrm{pH}$-dependent transcription regulator PacC as well as components of the lightregulated velvet complex. However, the similar expression profile of $R$. collo-cygni PacC (Fig. 6A) with SM gene transcription in $R$. collo-cygni does not conclusively indicate that this gene is involved in regulating SM gene expression in R. collo-cygni. The observation that PacC-specific 5'-GCCARG-3' binding sites (Tilburn et al. 1995) were present within 1,000 bp of the start codon of all the tested $R$. collo-cygni PKS genes (data not shown), except $P k s 3$, which has a slightly different expression profile, provides further evidence that may indicate that PacC could be involved in PKS core gene regulation in $R$. collocygni. Further studies on the possible role of $\mathrm{PacC}$ and other well-characterized fungal SM gene regulators are required to ascertain the true role of such genes in SM gene transcription in R. collo-cygni.

The velvet complex differentially regulates fungal SM biosynthesis in a light-dependent manner (Wiemann et al. 2010). This complex, which is formed by the three proteins VelB, VeA, and LaeA, is involved in regulating SM-related gene expression (Bayram et al. 2008). Most of the R. collo-cygni core genes examined showed higher transcript levels at the earliest stages of fungal colonization concomitant with higher levels of $\mathrm{VeA}$ and LaeA transcription (Fig. 6B). Similar results were found in other fungal species such as A. nidulans, F. fujikuroi, $F$. oxysporum, and B. maydis, where the velvet complex regulates SM biosynthesis (López-Berges et al. 2013; Park et al. 2012; Wiemann et al. 2010; Wu et al. 2012). These data indicate that the velvet complex may be involved in SM gene expression regulation in $R$. collo-cygni; however, the elevated transcription of $V e A$ and $L a e A$ at $10 \mathrm{dpi}$, when transcription of most $R$. collo-cygni SM core genes was declining, suggests that either control of secondary metabolism in $R$. collo-cygni may be under the control of other transcriptional regulators or the velvet complex may have another function in $R$. collo-cygni. Obtaining $R$. collo-cygni mutants impaired in LaeA or VeA expression may provide useful insights into the role played by the velvet complex in this fungus.

The two NR-PKS $P k s 2$ and $P k s 1$, with the highest sequence similarity to D. septosporum PksA, the core gene involved in dothistromin biosynthesis, and $P k s 5$, which was identified in a betaenone-like cluster (Fig. 3), were most abundant at 5 and 7 dpi, suggesting that, if the SM synthesized by these PKS are phytotoxins, then these SM could be produced early during $R$. collo-cygni colonization and stored. A red compound was observed inside vesicles within $R$. collo-cygni hyphae, indicating that metabolites such as rubellin could be stored within the fungus until needed (McGrann et al. 2016). However, the presence of SM in vesicles does not necessarily correspond to a storage phase. In A. parasiticus, several steps in the biosynthesis of the highly toxic SM aflatoxin are carried out in specialized vesicles termed aflatoxisomes (Chanda et al. 2009). Therefore, the previous observations of red-colored compounds stored in vesicles within $R$. collo-cygni hyphae may correspond to a specific phase in $R$. collo-cygni SM biosynthesis rather than a storage phase. Alternatively, the necrotic lesions observed as early as 7 dpi (Fig. 4) during RLS development indicate the action of a cell-death inducer. It is possible that it is these earlycolonization phase cell-death inducers that are being observed in hyphal vesicles although, because cell death can be induced through a number of pathways, it is unclear whether the observed cell death is linked with the action of a phytotoxin, SM, or some other product.

Transcript levels of the three HPS identified were also the highest during the early stages of colonization. If the chaetoglobosin A-like cluster located at the Hps2 locus is functional, the expression profile of Hps 2 suggests that this HPS may synthesize an antifungal agent early during the infection of the host plant. Considering the slow-growing nature of $R$. collocygni (Kaczmarek et al. 2017), which initially develops as an endophyte, chaetoglobosin production could act to eliminate fungi competing for the same ecological niche as $R$. collocygni. In vitro antifungal activity of $R$. collo-cygni was observed against common fungal pathogens of barley (Fig. 7). The antimicrobial activity of rubellin toxins produced by $R$. collo-cygni is reported by Miethbauer et al. (2008) as being light dependent. The assay used here was undertaken in constant darkness, suggesting that the antimicrobial effect of $R$. collo-cygni on in vitro fungal growth may not be linked with rubellin activity. Therefore, other SM such as chaetoglobosin or other non-SM compounds may be responsible for the observed antifungal activity and function to confer an ecological advantage to $R$. collo-cygni. Obtaining $R$. collo-cygni mutants in which SM core genes have been deleted may provide useful insights into the role played by SM in the biology of this fungus.

In silico analysis of the $R$. collo-cygni genome reveals that this fungus, like many other members of class Dothideomycetes, has the genetic potential to produce several classes of SM, including hybrid polyketide/nonribosomal peptides such as chaetoglobosin or polyketides such as betaenones or the previously characterized anthraquinone rubellin (Heiser et al. 2004; Miethbauer et al. 2003). Although the identification of 
the complete $R$. collo-cygni secondary metabolome may be difficult because several SM may only be produced under specific conditions relating to their unique functions in the biology of this fungus, our work suggests that $R$. collo-cygni has the genetic architecture to produce several SM potentially involved in the infection process. Therefore, characterizing the SM produced by $R$. collo-cygni during disease development would open new opportunities to understand the interaction between the fungus and its environment.

\section{MATERIALS AND METHODS}

\section{In silico identification}

of $\boldsymbol{R}$. collo-cygni secondary metabolism core genes.

The genome of $R$. collo-cygni isolate DK05 Rcc001, collected from the susceptible spring barley cultivar Braemar in Denmark in 2005, was recently sequenced and estimated to be $30.3 \mathrm{Mb}$ with 90x coverage and 11,617 predicted gene models (McGrann et al. 2016). RNAseq data were generated from total RNA extracted from mycelium of DK05 grown in vitro on PDA plates for 10 to 12 days and confirmed the expression of 8,514 of the predicted 11,617 genes under these conditions. A database containing the corresponding $11,617 \mathrm{R}$. collo-cygni protein models was created in GeneiousR9 software (Biomatters Ltd., Auckland, New Zealand). Protein sequences of PKS and NRPS involved in the synthesis of model fungal SM were downloaded from the National Center for Biotechnology Information (NCBI) GenBank database (Supplementary Table S6). These sequences were used to interrogate the $R$. collocygni genome using protein BLAST (BLASTp) (Altschul et al. 1990 ) to identify putative homologs in $R$. collo-cygni. Significant $R$. collo-cygni matches were retained if the BLASTp search with at least one of the fungal query proteins had an $e$ value $<1 . \mathrm{e}^{-10}$. The protein sequence of the corresponding $R$. collo-cygni gene models was then used to interrogate InterproScan and the PKS-NRPS analysis website to identify typical PKS or NRPS domains. Results from these analyses were combined and proteins that did not contain at least one of the PKS or NRPS domains were eliminated. Genes encoding the selected proteins were positioned on contigs or scaffolds within in the $R$. collo-cygni genome assembly using tBLASTn. For comparison purposes, the SM core gene complement of the $R$. collo-cygni genome was also analyzed using the programs AntiSMASH and SMURF.

\section{Identification of putative secondary metabolism clusters.}

To identify putative SM clusters, in silico genome walking was carried out both up- and downstream along $R$. collo-cygni contigs or scaffolds where each core gene was located. StarOrf (Massachusetts Institute of Technology, Cambridge, MA, U.S. A.) was used to determine open reading frames (ORF) along each contig or scaffold, with a minimal ORF size of $120 \mathrm{bp}$. The protein sequences of each ORF predicted in the genetic space surrounding each putative core gene were used to interrogate the NCBI nonredundant protein database with BLASTp with an $e$-value cut-off $<1 . \mathrm{e}^{-10}$. Each protein sequence of the best-match fungal BLAST hit was downloaded from GenBank and used to interrogate the R. collo-cygni protein models by BLASTp and the annotation of each gene in every cluster was confirmed. Borders of a gene cluster were delineated by a $5-\mathrm{kb}$ region of noncoding DNA or when three consecutive genes with no predicted function associated with SM production were identified. Comparative analysis of predicted $R$. collo-cygni SM clusters with SM clusters in other fungal species was performed with the AntiSMASH website (Blin et al. 2017) and similarity between genes within clusters was confirmed for each gene by reciprocal BLASTp.

\section{Phylogenetic analysis}

of $R$. collo-cygni PKS and HPS proteins.

Protein sequences of PKS and HPS involved in the biosynthesis of characterized fungal SM used in previous phylogenetic studies (Collemare et al. 2014; Fisch 2013) as well as sequences from fungi related to $R$. collo-cygni showing sequence similarity, based on BLASTp, to known SM core genes were downloaded from the GenBank database (Supplementary Table S7). Full-length sequences of PKS and HPS were aligned using MAFFT, and poorly aligned sequences were manually removed in Aliview (Larsson 2014). Phylogenetic trees were built using the RAxML, version 8.2.4 (Stamatakis et al. 2005), with 100 rapid bootstrap replicates and the protein model PROTGAMMAAUTO, where model testing is part of the algorithm.

\section{R. collo-cygni inoculation of barley seedlings.}

The RLS-susceptible spring barley cultivar Century was grown until growth stage 12/13 (Zadoks et al. 1974) in a controlled environment cabinet (Snijders Scientific, Tilburg, The Netherlands) at $18^{\circ} \mathrm{C}$, with a photoperiod of $16 \mathrm{~h}$ of light and $8 \mathrm{~h}$ of darkness supplemented with light at $250 \mu \mathrm{mol} \mathrm{m} \mathrm{m}^{-2} \mathrm{~s}^{-1}$ and $80 \%$ relative humidity. $R$. collo-cygni isolate Rcc001 DK05 ss2 was maintained and inoculum prepared as previously described (Makepeace et al. 2008; Peraldi et al. 2014). Inoculum was applied at a rate of $0.5 \mathrm{ml} / \mathrm{seedling}$, as previously reported (Makepeace et al. 2008). Inoculated plants were placed in a plastic tray ( 50 by 40 by $5 \mathrm{~cm}$ ), covered with a clear plastic lid to ensure maximum humidity, and incubated in the dark for $48 \mathrm{~h}$ at $18^{\circ} \mathrm{C}$. Following incubation, the light regime was restored and, $72 \mathrm{~h}$ later, the clear plastic lids were removed. Development of RLS was assessed at 5, 7, 10, 12, 15, 18, and 21 dpi. Visual assessment was carried out on the prophyll leaf and disease severity was estimated as the percentage of the leaf area covered by RLS lesions. The area under the disease progress curve (Shaner and Finney 1977) was calculated to report the development of the disease over time. GLA retention was also visually assessed at each time point because RLS development has been associated with leaf senescence (Schützendübel et al. 2008). Data show the mean \pm standard error calculated on six independent inoculation experiments, each of them containing two biological replications of 20 leaves each.

\section{Microscopic assessment of $\boldsymbol{R}$. collo-cygni colonization.}

Colonization by $R$. collo-cygni during RLS development was followed using light microscopy (Leica DMRBE; Leica Microsystems, Wetlzar, Germany). Leaves were harvested at 5, $7,10,12,15,18$, and $21 \mathrm{dpi}$ and cleared in $70 \%$ ethanol for 7 days prior to staining with $0.05 \%$ trypan blue in lacto-glycerol and briefly destaining with $100 \%$ ethanol. For each leaf, a minimum of 120 stomata were observed over its full length. Fungal development stages and the associated host response was scored in three categories based on those described previously (McGrann and Brown 2018): (i) proportion of stomata penetrated by fungal hyphae, (ii) proportion of stomata surrounded by necrosis, and (iii) the proportions of stomata with fungal conidiophore-like structures emerging. Data show the mean \pm standard error calculated on six independent experiments, each of them containing two biological replications of two leaves each.

\section{$R$. collo-cygni DNA quantification during RLS development.}

Two leaves were harvested from Century plants at each time point between 5 and 21 dpi and snap frozen in liquid nitrogen, and DNA was extracted using the DNeasy Plant Mini Kit (Qiagen Ltd., Hilden, Germany). R. collo-cygni DNA 
quantification based on an internal transcribed spacer region was carried out using qPCR as described by Taylor et al. (2010). Each reaction volume of $25 \mu$ consisted of $1 \times$ iQ Supermix (Bio-Rad, Hercules, CA), forward primer RamF6 $\left(5^{\prime}\right.$-CGTCATTTCACCACTCAAG-3') and reverse primer RamR6 (5'-CCTCTGCGAATAGTTGCC-3') at 400 nmol lit$\mathrm{er}^{-1}, 5 \mu \mathrm{l}$ of DNA template $\left(5 \mathrm{ng} \mu^{-1}\right), R$. collo-cygni probe Ram6 (5' - GCGATTCCGGCTGAGCGGTTCGTCATCGCG-3') at $150 \mathrm{nmol} \mathrm{liter}^{-1}$, and molecular-grade water to the final volume. Reactions were performed in a Mx3000P thermocycler (Agilent Technologies, Santa Clara, CA) using the following program: initial denaturation of $10 \mathrm{~min}$ at $95^{\circ} \mathrm{C}$; followed by 50 cycles of $95^{\circ} \mathrm{C}$ for $20 \mathrm{~s}, 55^{\circ} \mathrm{C}$ for $20 \mathrm{~s}$, and $72^{\circ} \mathrm{C}$ for $20 \mathrm{~s}$; followed by a final extension of $95^{\circ} \mathrm{C}$ for $1 \mathrm{~min}$. Potential primer-dimer formation and confirmation of single genespecific product amplification was monitored at the end of each reaction by melt curve analysis. Data show the mean quantity (in picograms) of $R$. collo-cygni DNA calculated from a standard curve produced by a 10-fold dilution series of $R$. collo-cygni DNA. $R$. collo-cygni DNA levels were assessed in five independent inoculation experiments.

\section{Expression profiling of $\boldsymbol{R}$. collo-cygni SM-related genes.}

Transcript levels of putative $R$. collo-cygni SM-related genes were assessed as RLS developed 5 to 21 dpi with Rcc001 DK05 ss2. For each biological replicate, two leaves were harvested and pooled prior to snap freezing in liquid nitrogen. Total RNA was extracted using the RNeasy Mini Kit (Qiagen Ltd.) following the manufacturer's instructions. Contaminating genomic DNA was removed from RNA samples using TURBO DNase I (Ambion, Thermo Fisher Scientific, Austin, TX). cDNA was synthesized from $1 \mu \mathrm{g}$ of total RNA using the SuperScript III First-Strand Synthesis System (Invitrogen, Carlsbad, CA). cDNA was diluted 10-fold in sterile distilled water and used in qRT-PCR using the SybrGreen Jump Start Taq system (Sigma, Dorset, U.K.) and a Mx3000P thermocycler (Agilent Technologies). The stability of four $R$. collo-cygni reference genes-elongation factor 1- $\alpha$; glyceraldehyde-3phosphate dehydrogenase (GAPDH), $\alpha$-tubulin, and actin-was assessed under the conditions of this experiment using GeNorm (Vandesompele et al. 2002). The two most stable reference genes (GAPDH and $\alpha$-tubulin) were used for cDNA normalization. Gene-specific primers (Supplementary Table S8) for $R$. collocygni genes of interest were designed using Primer3. Primers with amplification efficiency ranging from 80 to $110 \%$ were used for transcript quantification based on the $\mathrm{E}^{-\Delta \Delta \mathrm{Ct}}$ method (Livak and Schmittgen 2001). Data show the mean normalized expression values \pm standard error of three independent experiments, each of them containing two biological replicates.

\section{In vitro fungal growth inhibition assays.}

Growth inhibition of commonly occurring barley pathogens F. poae, F. graminearum, Pyrenophora teres, and Rhynchosporium commune by Ramularia collo-cygni isolate Rcc001 DK05 ss2 was assessed in dual-culture plate assays. The assay was carried out in 9-cm Petri dishes (Sarstedt, Nümbrecht, Germany), on two different media, AEA and PDA (Sigma) containing streptomycin at $5 \mu \mathrm{g} \mathrm{ml}^{-1}$. Growth inhibition of Rhynchosporium commune was only tested on PDA due to the slow growing nature of both Rhynchosporium commune and $R$. collo-cygni on AEA. To compensate for the differential in vitro growth rate between $R$. collo-cygni and $F$. poae, $F$. graminearum, and $P$. teres, an 8-mm plug of $R$. collo-cygni mycelium was extracted from a 14-day-old culture plate, placed $2 \mathrm{~cm}$ from the border of the Petri dish, and allowed to grow for 14 days in isolation. Mycelial plugs from competing fungal species were taken from 14-day-old cultures and placed $3 \mathrm{~cm}$ away from the $R$. collo-cygni culture. Considering the slow-growing nature of both $R$. collo-cygni and Rhynchosporium commune, the mycelial plugs of the two fungi were added to the culture plate at the same time and placed $3 \mathrm{~cm}$ apart. All fungal cultures were grown in a Sanyo MIR-254 incubator (Sanyo Electric Ltd., Osaka, Japan) in constant darkness at $15^{\circ} \mathrm{C}$. Fungal radial growth was measured after 10 days for $P$. teres and $F$. poae, 7 days for $F$. graminearum, and 21 days for Rhynchosporium commune. The assay was repeated at least three times for each fungus, with experimental replicates consisting of at least three individual plates per assay.

\section{Data analysis.}

All statistical tests were performed in GenStat, v.16 (Payne et al. 2009). Data were analyzed by analysis of variance using generalized linear models (GLM) unless stated otherwise. Each GLM assessed how the various factors that contributed to the design of the different experiments affected variation in the scored phenotype. Disease score data from the $R$. collo-cygni time-course inoculation experiment were Logit+ transformed (McGrann et al. 2014) prior to analysis. GLA retention during RLS development was assessed with a linear mixed model of repeated measures using the uniform correlation/split plot in time covariance matrix (McGrann et al. 2015a). The factors day, experiment, and tray were fixed in the model whereas the leaf-day interaction term was a random factor. The microscopy experiment was analyzed using a GLM where each score category was analyzed separately as a proportion of the total number of stomata scored on each leaf using a binomial distribution as the link function. Growth inhibition effects of $R$. collo-cygni on each pathogen were assessed separately using a GLM assessing variation attributable to experimental replicates and the direction of fungal growth from the point of inoculation either toward the $R$. collo-cygni culture or toward the edge of the culture plate. Data are presented as growth inhibition calculated as the ratio (percentage) of radial fungal growth toward $R$. collo-cygni culture from point of inoculation/radial fungal growth toward the edge of the culture plate from point of inoculation. Growth on either PDA or AEA was assessed separately. After modeling, treatments were compared within each experiment using $t$ test probabilities and applying Fisher's least significant difference to unplanned comparisons.

\section{LITERATURE CITED}

Alkan, N., Friedlander, G., Ment, D., Prusky, D., and Fluhr, R. 2015. Simultaneous transcriptome analysis of Colletotrichum gloeosporioides and tomato fruit pathosystem reveals novel fungal pathogenicity and fruit defense strategies. New Phytol. 205:801-815.

Altschul, S. F., Gish, W., Miller, W., Meyers, E. W., and Lipman, D. J. 1990. Basic local alignment research tool. J. Mol. Biol. 215:403-410.

Awakawa, T., Yokota, K., Funa, N., Doi, F., Mori, N., Watanabe, H., and Horinouchi, S. 2009. Physically discrete beta-lactamase-type thioesterase catalyzes product release in atrochrysone synthesis by iterative type I polyketide synthase. Chem. Biol. 16:613-623.

Bayram, Ö., Krappmann, S., Ni, M., Bok, J. W., Helmstaedt, K., Yu, J., and Braus, G. H. 2008. VelB/VeA/LaeA complex coordinates light signal with fungal development and secondary metabolism. Science 320: 1504-1506.

Blin, K., Wolf, T., Chevrette, M. G., Lu, X., Schwalen, C. J., Kautsar, S. A., Suarez Duran, H. G., De Los Santos, E. L. C., Kim, H. U., Nave, M., Dickschat, J. S., Mitchell, D. A., Shelest, E., Breitling, R., Takano, E., Lee, S. Y., Weber, T., and Medema, M. H. 2017. AntiSMASH 4.0-Improvements in chemistry prediction and gene cluster boundary identification. Nucleic Acids Res. 45:W36-W41.

Bradshaw, R. E., Guo, Y., Sim, A. D., Kabir, M. S., Chettri, P., Ozturk, I. K., Hunziker, L., Ganley, R. J., and Cox, M. P. 2016. Genome-wide gene expression dynamics of the fungal pathogen Dothistroma septosporum throughout its infection cycle of the gymnosperm host Pinus radiata. Mol. Plant Pathol. 17:210-224. 
Brakhage, A. A. 2013. Regulation of fungal secondary metabolism. Nat. Rev. Microbiol. 11:21-32.

Bushley, K. E., and Turgeon, B. G. 2010. Phylogenomics reveals subfamilies of fungal nonribosomal peptide synthetases and their evolutionary relationships. BMC Evol. Biol. 10:26.

Cairns, T., and Meyer, V. 2017. In silico prediction and characterization of secondary metabolite biosynthetic gene clusters in the wheat pathogen Zymoseptoria tritici. BMC Genomics 18:631.

Calvo, A. M., Wilson, R. A., Bok, J. W., and Keller, N. P. 2002. Relationship between secondary metabolism and fungal development. Microbiol. Mol. Biol. Rev. 66:447-459.

Chanda, A., Roze, L. V., Kang, S., Artymovich, K. A., Hicks, G. R., Raikhel, N. V., Calvo, A. M., and Linz, J. E. 2009. A key role for vesicles in fungal secondary metabolism. Proc. Natl. Acad. Sci. USA 106: 19533-19538.

Chiang, Y. M., Szewczyk, E., Davidson, A. D., Entwistle, R., Keller, N. P., Wang, C. C. C., and Oakley, B. R. 2010. Characterization of the Aspergillus nidulans monodictyphenone gene cluster. Appl. Environ. Microbiol. 76:2067-2074.

Choquer, M., Dekkers, K. L., Chen, H., Cao, L., Ueng, P. P., Daub, M. E., and Chung, K. 2005. The CTB1 gene encoding a fungal polyketide synthase is required for cercosporin biosynthesis and fungal virulence of Cercospora nicotianae. Mol. Plant-Microbe Interact. 18:468-476.

Collemare, J., Griffiths, S., Iida, Y., Karimi Jashni, M., Battaglia, E., Cox, R. J., and de Wit, P. J. G. M. 2014. Secondary metabolism and biotrophic lifestyle in the tomato pathogen Cladosporium fulvum. PLoS One 9:e85877.

Cromey, M. G., Harvey, I. C., Sheridan, J. E., and Grbavac, N. 2002. Occurrence, importance, and control of Ramularia collo-cygni in NewZealand. Pages 337-342 in: Proc. 2nd Int. Workshop Barley Leaf Blights, A. Yahyaoui, L. Brader, A. Tekauz, H. Wallwork, and B. Steffenson, eds. Aleppo, Syria.

Crous, P., Kang, J.-C., and Braun, U. 2001. A phylogenetic redefinition of anamorph genera in Mycosphaerella based on ITS rDNA sequence and morphology. Mycologia 93:1081-1101.

de Wit, P. J. G. M., van der Burgt, A., Ökmen, B., Stergiopoulos, I., AbdElsalam, K. A., Aerts, A. L., Bahkali, A. H., Beenen, H. G., Chettri, P., Cox, M. P., Datema, E., de Vries, R. P., Dhillon, B., Ganley, A. R., Griffiths, S. A., Guo, Y., Hamelin, R. C., Henrissat, B., Kabir, M. S., Jashni, M. K., Kema, G., Klaubauf, S., Lapidus, A., Levasseur, A., Lindquist, E., Mehrabi, R., Ohm, R. A., Owen, T. J., Salamov, A., Schwelm, A., Schijlen, E., Sun, H., van den Burg, H. A., van Ham, R. C. H. J., Zhang, S., Goodwin, S. B., Grigoriev, I. V., Collemare, J., and Bradshaw, R. E. 2012. The genomes of the fungal plant pathogens Cladosporium fulvum and Dothistroma septosporum reveal adaptation to different hosts and lifestyles but also signatures of common ancestry. PLoS Genet. 8:e1003088.

Fisch, K. M. 2013. Biosynthesis of natural products by microbial iterative hybrid PKS-NRPS. RSC Advances 3:18228-18247.

Gan, P., Ikeda, K., Irieda, H., Narusaka, M., O’Connell, R. J., Narusaka, Y., Takano, Y., Kubo, Y., and Shirasu, K. 2013. Comparative genomic and transcriptomic analyses reveal the hemibiotrophic stage shift of Colletotrichum fungi. New Phytol. 197:1236-1249.

Gardiner, D. M., Jarvis, R. S., and Howlett, B. J. 2005. The ABC transporter gene in the sirodesmin biosynthetic gene cluster of Leptosphaeria maculans is not essential for sirodesmin production but facilitates selfprotection. Fungal Genet. Biol. 42:257-263.

Griffiths, S., Mesarich, C. H., Saccomanno, B., Vaisberg, A., de Wit, P. J. G. M., Cox, R., and Collemare, J. 2016. Elucidation of cladofulvin biosynthesis reveals a cytochrome P450 monooxygenase required for anthraquinone dimerization. Proc. Natl. Acad. Sci. USA 113:6851-6856.

Havis, N. D., Brown, J. K. M., Clemente, G., Frei, P., Jedryczka, M., Kaczmarek, J., Kaczmarek, M., Matusinsky, P., McGrann, G. R. D., Piotrowska, M., Sghyer, H., Tellier, A., and Hess, M. 2015. Ramularia collo-cygni-An emerging pathogen of barley crops. Phytopathology 105:895-904.

Havis, N. D., Nyman, M., and Oxley, S. J. P. 2013. Evidence for seed transmission and symptomless growth of Ramularia collo-cygni in barley (Hordeum vulgare). Plant Pathol. 63:929-936.

Heiser, I., Heß, M., Schmidtke, K.-U., Vogler, U., Miethbauer, S., and Liebermann, B. 2004. Fatty acid peroxidation by rubellin B, C and D, phytotoxins produced by Ramularia collo-cygni (Sutton et Waller). Physiol. Mol. Plant Pathol. 64:135-143.

Heiser, I., Sachs, E., and Liebermann, B. 2003. Photodynamic oxygen activation by rubellin $\mathrm{D}$, a phytotoxin produced by Ramularia collocygni (Sutton et Waller). Physiol. Mol. Plant Pathol. 62:29-36.

Huss, H. 2002. The biology of Ramularia collo-cygni. Pages 321-328 in: Proc. 2nd Int. Workshop Barley Leaf Blights, A. Yahyaoui, L. Brader, A. Tekauz, H. Wallwork, and B. Steffenson, eds. Aleppo, Syria.
Ichihara, A., Oikawa, H., Hayashi, K., and Sakamura, S. 1983. Structures of betaenones A and B, novel phytotoxins from Phoma betae Fr. J. Am. Chem. Soc. 105:2907-2908.

Kabir, M. S., Ganley, R. J., and Bradshaw, R. E. 2015. Dothistromin toxin is a virulence factor in Dothistroma needle blight of pines. Plant Pathol. 64:225-234.

Kaczmarek, M., Piotrowska, M. J., Fountaine, J. M., Gorniak, K., McGrann, G. R. D., Armstrong, A., Wright, K. M., Newton, A. C., and Havis, N. D. 2017. Infection strategy of Ramularia collo-cygni and development of Ramularia leaf spot on barley and alternative graminaceous hosts. Plant Pathol. 66:45-55.

Keon, J., Rudd, J. J., Antoniw, J., Skinner, W., Hargreaves, J., and Hammond-Kosack, K. 2005. Metabolic and stress adaptation by Mycosphaerella graminicola during sporulation in its host revealed through microarray transcription profiling. Mol. Plant Pathol. 6:527-540.

Kroken, S., Glass, N. L., Taylor, J. W., Yoder, O. C., and Turgeon, B. G. 2003. Phylogenomic analysis of type I polyketide synthase genes in pathogenic and saprobic Ascomycetes. Proc. Natl. Acad. Sci. USA100: $15670-15675$.

Larsson, A. 2014. AliView: A fast and lightweight alignment viewer and editor for large datasets. Bioinformatics 30:3276-3278.

Liao, H.-L., and Chung, K.-R. 2008. Genetic dissection defines the roles of elsinochrome Phytotoxin for fungal pathogenesis and conidiation of the citrus pathogen Elsinoë fawcettii. Mol. Plant-Microbe Interact. 21:469-479.

Lim, F. Y., Hou, Y., Chen, Y., Oh, J. H., Lee, I., Bugni, T. S., and Keller, N. P. 2012. Genome-based cluster deletion reveals an endocrocin biosynthetic pathway in Aspergillus fumigatus. Appl. Environ. Microbiol. 78:4117-4125.

Livak, K. J., and Schmittgen, T. D. 2001. Analysis of relative gene expression data using real-time quantitative PCR and the $2^{-\Delta \Delta C T}$ method. Methods 25:402-408.

López-Berges, M. S., Hera, C., Sulyok, M., Schäfer, K., Capilla, J., Guarro, J., and Di Pietro, A. 2013. The velvet complex governs mycotoxin production and virulence of Fusarium oxysporum on plant and mammalian hosts. Mol. Microbiol. 87:49-65.

Lorang, J. M., Carkaci-Salli, N., and Wolpert, T. J. 2004. Identification and characterization of victorin sensitivity in Arabidopsis thaliana. Mol Plant-Microbe Interact. 17:577-582.

Makepeace, J. C., Havis, N. D., Burke, J. I., Oxley, S. J. P., and Brown, J. K. M. 2008. A method of inoculating barley seedlings with Ramularia collo-cygni. Plant Pathol. 57:991-999.

McGrann, G. R. D., Andongabo, A., Sjökvist, E., Trivedi, U., Dussart, F., Kaczmarek, M., Mackenzie, A., Fountaine, J. M., Taylor, J. M. G., Paterson, L. J., Gorniak, K., Burnett, F., Kanyuka, K., HammondKosack, K. E., Rudd, J. J., Blaxter, M., and Havis, N. D. 2016. The genome of the emerging barley pathogen Ramularia collo-cygni. BMC Genomics 17:584.

McGrann, G. R. D., and Brown, J. K. M. 2018. The role of reactive oxygen in the development of Ramularia leaf spot disease in barley seedlings. Ann. Bot. 121:415-430.

McGrann, G. R. D., and Havis, N. D. 2017. Ramularia leaf spot: A newly important threat to barley production. Outlooks Pest Manage. 28:65-69.

McGrann, G. R. D., Stavrinides, A., Russell, J., Corbitt, M. M., Booth, A., Chartrain, L., Thomas, W. T. B., and Brown, J. K. M. 2014. A trade off between mlo resistance to powdery mildew and increased susceptibility of barley to a newly important disease, Ramularia leaf spot. J. Exp. Bot. 65:1025-1037.

McGrann, G. R. D., Steed, A., Burt, C., Goddard, R., Lachaux, C., Bansal, A., Corbitt, M., Gorniak, K., Nicholson, P., and Brown, J. K. M. 2015 a. Contribution of the drought tolerance-related stress-responsive NAC1 transcription factor to resistance of barley to Ramularia leaf spot. Mol. Plant Pathol. 16:201-209.

McGrann, G. R. D., Steed, A., Burt, C., Nicholson, P., and Brown, J. K. M. 2015b. Differential effects of lesion mimic mutants in barley on disease development by facultative pathogens. J. Exp. Bot. 66:3417-3428.

Miethbauer, S., Günther, W., Schmidtke, K.-U., Heiser, I., Gräfe, S., Gitter, B., and Liebermann, B. 2008. Uredinorubellins and caeruleoramularin, photodynamically active anthraquinone derivatives produced by two species of the genus Ramularia. J. Nat. Prod. 71:1371-1375.

Miethbauer, S., Haase, S., Schmidtke, K.-U., Günther, W., Heiser, I., and Liebermann, B. 2006. Biosynthesis of photodynamically active rubellins and structure elucidation of new anthraquinone derivatives produced by Ramularia collo-cygni. Phytochemistry 67:1206-1213.

Miethbauer, S., Heiser, I., and Liebermann, B. 2003. The phytopathogenic fungus Ramularia collo-cygni produces biologically active rubellins on infected barley leaves. J. Phytopathol. 151:665-668.

Muria-Gonzalez, M. J., Chooi, Y. H., Breen, S., and Solomon, P. S. 2015. The past, present and future of secondary metabolite research in the Dothideomycetes. Mol. Plant Pathol. 16:92-107. 
Navarre, D. A., and Wolpert, T. J. 1999. Victorin induction of an apoptotic/senescence-like response in oats. Plant Cell 11:237-249.

Noar, R. D., and Daub, M. E. 2016. Bioinformatics prediction of polyketide synthase gene clusters from Mycosphaerella fijiensis. PLoS One 11: e0158471.

O'Connell, R. J., Thon, M. R., Hacquard, S., Amyotte, S. G., Kleemann, J., Torres, M. F., Damm, U., Buiate, E. A., Epstein, L., Alkan, N., Altmüller, J., Alvarado-Balderrama, L., Bauser, C. A., Becker, C., Birren, B. W. Chen, Z., Choi, J., Crouch, J. A., Duvick, J. P., Farman, M. A., Gan, P., Heiman, D., Henrissat, B., Howard, R. J., Kabbage, M., Koch, C., Kracher, B., Kubo, Y., Law, A. D., Lebrun, M.-H., Lee, Y.-H., Miyara, I., Moore, N., Neumann, U., Nordström, K., Panaccione, D. G., Panstruga, R., Place, M., Proctor, R. H., Prusky, D., Rech, G., Reinhardt, R., Rollins, J. A., Rounsley, S., Schardl, C. L., Schwartz, D. C., Shenoy, N., Shirasu, K., Sikhakolli, U. R., Stüber, K., Sukno, S. A., Sweigard, J. A., Takano, Y., Takahara, H., Trail, F., van der Does, H. C., Voll, L. M., Will, I., Young, S., Zeng, Q., Zhang, J., Zhou, S., Dickman, M. B., SchulzeLefert, P., Ver Loren van Themaat, E., Ma, L.-J., and Vaillancourt, L. J. 2012. Lifestyle transitions in plant pathogenic Colletotrichum fungi deciphered by genome and transcriptome analyses. Nat. Genet. 44: 1060-1065.

Ohm, R. A., Feau, N., Henrissat, B., Schoch, C. L., Horwitz, B. A., Barry, K. W., Condon, B. J., Copeland, A. C., Dhillon, B., Glaser, F., Hesse, C. N., Kosti, I., LaButti, K., Lindquist, E. A., Lucas, S., Salamov, A. A., Bradshaw, R. E., Ciuffetti, L., Hamelin, R. C., Kema, G. H. J., Lawrence, C., Scott, J. A., Spatafora, J. W., Turgeon, B. G., de Wit, P. J. G. M., Zhong, S., Goodwin, S. B., and Grigoriev, I. V. 2012. Diverse lifestyles and strategies of plant pathogenesis encoded in the genomes of eighteen Dothideomycetes fungi. PLoS Pathog. 8:e1003037.

Ohtsubo, K., Saito, M., Sekita, S., Yoshihira, K., and Natori, S. 1978. Acute toxic effects of chaetoglobosin A, a new cytochalasan compound produced by Chaetomium globosum, on mice and rats. Jpn. J. Exp. Med. 48:105-110.

Palma-Guerrero, J., Torriani, S. F. F., Zala, M., Carter, D., Courbot, M., Rudd, J. J., McDonald, B. A., and Croll, D. 2016. Comparative transcriptomic analyses of Zymoseptoria tritici strains show complex lifestyle transitions and intraspecific variability in transcription profiles. Mol. Plant Pathol. 17:845-859.

Park, H. S., Ni, M., Jeong, K. C., Kim, Y. H., and Yu, J. H. 2012. The role, interaction and regulation of the Velvet regulator VelB in Aspergillus nidulans. PLoS One 7:e45935.

Payne, R., Murray, D., Harding, S., Baird, D., and Soutar, D. 2009. Page 256 in: GenStat for Windows, 12th ed. VSN International, Hemel Hempstead, U.K

Peraldi, A., Griffe, L. L., Burt, C., McGrann, G. R. D., and Nicholson, P. 2014. Brachypodium distachyon exhibits compatible interactions with Oculimacula spp. and Ramularia collo-cygni, providing the first pathosystem model to study eyespot and ramularia leaf spot diseases. Plant Pathol. 63:554-562.

Rudd, J. J., Kanyuka, K., Hassani-Pak, K., Derbyshire, M., Andongabo, A., Devonshire, J., Lysenko, A., Saqi, M., Desai, N. M., Powers, S. J., Hooper, J., Ambroso, L., Bharti, A., Farmer, A., Hammond-Kosack, K. E., Dietrich, R. A., and Courbot, M. 2015. Transcriptome and metabolite profiling of the infection cycle of Zymoseptoria tritici on wheat reveals a biphasic interaction with plant immunity involving differential pathogen chromosomal contributions and a variation on the hemibiotrophic lifestyle definition. Plant Physiol. 167:1158-1185.

Scherlach, K., Boettger, D., Remme, N., and Hertweck, C. 2010. The chemistry and biology of cytochalasans. Nat. Prod. Rep. 27:869-886.

Schümann, J., and Hertweck, C. 2007. Molecular basis of cytochalasan biosynthesis in fungi: Gene cluster analysis and evidence for involvement of a PKS-NRPS hybrid synthase by RNA silencing. J. Am. Chem. Soc. 129: 9564-9565

Schützendübel, A., Stadler, M., Wallner, D., and von Tiedemann, A. 2008. A hypothesis on physiological alterations during plant ontogenesis governing susceptibility of winter barley to Ramularia leaf spot. Plant Pathol. 57:518-526.

Shaner, G., and Finney, R. E. 1977. The effect of nitrogen fertilization on the expression of slow-mildewing resistance in Knox wheat. Phytopathology 67:1051-1056.

So, K.-K., Chung, Y.-J., Kim, J.-M., Kim, B.-T., Park, S.-M., and Kim, D.-H. 2015. Identification of a polyketide synthase gene in the synthesis of phleichrome of the phytopathogenic fungus Cladosporium phlei. Mol. Cells 38:1105-1110.

Stamatakis, A., Ludwig, T., and Meier, H. 2005. RAxML-III: A fast program for maximum likelihood-based inference of large phylogenetic trees. Bioinformatics 21:456-463.

Stergiopoulos, I., Collemare, J., Mehrabi, R., and De Wit, P. J. G. M. 2013. Phytotoxic secondary metabolites and peptides produced by plant pathogenic Dothideomycete fungi. FEMS Microbiol. Rev. 37:67-93.

Taylor, J. M. G., Paterson, L. J., and Havis, N. D. 2010. A quantitative realtime PCR assay for the detection of Ramularia collo-cygni from barley (Hordeum vulgare). Lett. Appl. Microbiol. 50:493-499.

Tilburn, J., Sarkar, S., Widdick, D. A., Espeso, E. A., Orejas, M., Mungroo, J., Peñalva, M. A., and Arst, H. N. 1995. The Aspergillus PacC zinc finger transcription factor mediates regulation of both acid- and alkalineexpressed genes by ambient pH. EMBO J. 14:779-790.

Ugai, T., Minami, A., Fujii, R., Tanaka, M., Oguri, H., Gomi, K., and Oikawa, H. 2015. Heterologous expression of highly reducing polyketide synthase involved in betaenone biosynthesis. Chem. Commun (Camb.) 51:1878-1881.

Vandesompele, J., De Preter, K., Pattyn, F., Poppe, B., Van Roy, N., De Paepe, A., and Speleman, F. 2002. Accurate normalization of real-time quantitative RT-PCR data by geometric averaging of multiple internal control genes. Genome Biol. 3:0034.1-0034.11.

Walters, D. R., Havis, N. D., and Oxley, S. J. P. 2008. Ramularia collo-cygni: The biology of an emerging pathogen of barley. FEMS Microbiol. Lett. 279:1-7.

Wiemann, P., Brown, D. W., Kleigrewe, K., Bok, J. W., Keller, N. P., Humpf, H. U., and Tudzynski, B. 2010. FfVel1 and FfLae1, components of a velvet-like complex in Fusarium fujikuroi, affect differentiation, secondary metabolism and virulence. Mol. Microbiol. 77:972-994.

Wu, D., Oide, S., Zhang, N., Choi, M. Y., and Turgeon, B. G. 2012. ChLae1 and ChVel1 regulate T-toxin production, virulence, oxidative stress response, and development of the maize pathogen Cochliobolus heterostrophus. PLoS Pathog. 8:e1002542.

Zadoks, J., Chang, T., and Konzak, C. 1974. A decimal code for the growth stages of cereals. Weed Res. 14:415-421.

Zhang, G., Zhang, Y., and Qin, J. 2013. Antifungal metabolites produced by Chaetomium globosum No.04, an endophytic fungus isolated from Ginkgo biloba. Indian J. Microbiol. 53:175-180.

\section{AUTHOR-RECOMMENDED INTERNET RESOURCES}

National Center for Biotechnology Information GenBank database: https://www.ncbi.nlm.nih.gov/genbank

InterproScan: https://www.ebi.ac.uk

PKS-NRPS analysis website: http://nrps.igs.umaryland.edu

AntiSMASH program: https://fungismash.secondarymetabolites.org

SMURF program: http://www.jcvi.org/smurf

StarOrf program: http://star.mit.edu/orf

Primer3: http://primer3.ut.ee

MAFFT: https://www.ebi.ac.uk 